\title{
Saliency metric for subadditive dissimilarity judgments of rectangles
}

\author{
ALEJANDRO A. LAZARTE and PETER H. SCHÖNEMANN \\ Purdue University, West Lafayette, Indiana
}

\begin{abstract}
Previous findings that dissimilarity judgments for rectangles are strongly subadditive, that qualitative individual differences are always present, and that four-parameter psychophysical maps can reproduce the average ratings (Schönemann \& Lazarte, 1987) are replicated. However, the present study in addition suggests that the metric for bounded response transformation, previously used to restore additivity of responses, has a modest effect on the fits of the psychophysical maps. The differential weighing of the coordinates already incorporates segmental subadditivity predictions into the maps. In fact, the psychophysical maps define a subadditive saliency metric that depends on assigning larger weights to larger coordinates. This constraint on the weights of these maps, together with the response times, allows us to identify a strategy shift when subjects respond to two classes of stimulus pairs: For rectangle-rectangle pairs, subjects center on the shape and size of the rectangles; for square-rectangle pairs, the focus is on height and width.
\end{abstract}

Several articles have been published in which direct dissimilarity ratings are related to geometric models of a psychological space (Attneave, 1950; Borg \& Leutner, 1983; Dunn, 1983; Hyman \& Well, 1967, 1968; Krantz \& Tversky, 1975; Shepard, 1964; Wender, 1971; WienerEhrlich, 1978). Following Attneave (1950), in these studies it has been assumed that observed dissimilarities are monotonically related to distances in a subjective dimensional geometric space. These distances are produced by the combination of the segments that stimulus pairs project onto the axes of that assumed "subjective" or "psychological" space.

A geometric model for dissimilarities basically assumes that any stimulus, $A$, can be characterized as having locations along several "attributes" or dimensions, thus $A=\left(a_{1}, a_{2}, \ldots a_{p}\right)$. Also, for any stimuli $A$ and $B$, a distance or metric function, $d(A, B)$, is defined such that $d$ satisfies at least:

$$
\text { Positivity: } \quad \begin{array}{r}
d(A, B)>d(A, A)=0, \\
\text { for } A \neq B,
\end{array}
$$

Symmetry: $\quad d(A, B)=d(B, A)$,

Triangle Inequality: $d(A, B)+d(B, C) \geq d(A, C)$.

Individuals produce dissimilarity judgments, $\delta(A, B)$, that are usually assumed to relate monotonically to the

\footnotetext{
We thank the assistance of Robert Melara and Helena Kadlec for their comments on previous drafts of this article. We also thank John C. Dunn, one of our reviewers, for suggesting Equation 18B, an alternative representation of the saliency metric, and an anonymous reviewer for helpful comments. Requests for reprints should be sent to Alejandro Lazarte, c/o Peter H. Schönemann, Department of .Psychological Sciences, Purdue University, West Lafayette, IN 47906
}

distances, $d(A, B)$, of the stimuli on that psychological space-that is, $\delta(A, B)=\Phi[d(A, B)]$, where $\Phi$ is strictly monotonic. A subset of distance functions is the Minkowski metrics family, defined as:

$$
d(A, B)=\left[\Sigma_{i}\left(\left|a_{i}-b_{i}\right|^{r}\right)\right]^{1 / r} \quad i=1,2, \ldots p,
$$

where $r>1$ and $\left|a_{i}-b_{i}\right|$ is the segment that the stimulus pair projects onto the $i$ th dimension. The two most popular members of this family are the Euclidean metric $(r=2)$ and the city-block metric $(r=1)$. All Minkowski metrics satisfy segmental additivity, which implies that for any stimuli $A$ and $B$ that differ on exactly one dimension, there exists a stimulus $C$ such that

$$
d(A, C)+d(C, B)=d(A, B) .
$$

The fit of a dimensional geometric model to the data depends on the orientation of the coordinate system, the metric defined over this system, and the monotonic transformation that is assumed to relate the observed dissimilarities to the distances. All three aspects are highly interconnected; to study any one of them requires a working definition of the other two. Psychologically, the orientation problem asks which stimulus attributes are used by the subjects to produce a response, while the metric problem asks how the projections of the stimuli onto the coordinate axis are combined to produce an overall response.

\section{The Dimensional Orientation Problem}

Among the most commonly used stimuli to obtain direct dissimilarity ratings are simple geometric figures (e.g., parallelograms, rectangles, or "circle and spoke" stimuli used by Shepard, 1964) and Munsell color patches. Rectangles can be considered as located along height $(H)$ and width $(W)$ coordinates, or along another set of axes related to height and width, area $(A)$ and shape $(S)$. In the literature, three pairs of axes generally describe the physical 
design configuration for rectangles: (1) $H / W$, direct height and width (Borge \& Leutner, 1983; Dunn, 1983; Schönemann, Dorcey, \& Kienapple, 1985; Schönemann \& Lazarte, 1987); (2) $A / S$, area (height $\times$ width) and shape (height/width) (Wender, 1971); and (3) "orthogonal" configurations on $\log$ height versus $\log$ width and log area versus log shape (Krantz \& Tversky, 1975; Wiener-Ehrlich, 1978).

Even with such simple stimuli, the determination of the orientation of the dimensions is problematic. Some authors have argued for an area/shape configuration where both dimensions interact (Krantz \& Tversky, 1975; Wender, 1971). However, although this particular interaction was shown later to be an artifact of the physical stimuli design (Schönemann, 1977), others have reported an interaction effect in the height-width coordinate system (Wiener-Ehrlich, 1978). Still other studies have shown no interaction in this height-width configuration (Borg \& Leutner, 1983).

In most of the studies above, an implicit assumption is that all subjects will use the same coordinate system. In practice, the perceived orientation of the stimulus configuration is inferred after applying a nonmetric multidimensional scaling (MDS) algorithm to the group average dissimilarities. Some researchers (Krantz \& Tversky, 1975) have used MDS models that allow individual differences through differential weighing of the dimensions (Carrol \& Chang, 1970; Horan, 1969; Schönemann, 1972). However, these models also assume that all subjects use the same dimensions.

More recently, there has been growing evidence that different people judge dissimilarities between rectangles differently. Schönemann and collaborators (Schönemann et al., 1985; Schönemann \& Lazarte, 1987), using the actual, untransformed, individual dissimilarity ratings, found consistent individual differences in the rating strategies employed by their subjects. Even with the same physical configuration (height and width in this case), some subjects used height and width, others shape and area, and still others shape only. In one study (Schönemann \& Lazarte, 1987), a completely crossed height-width physical design was employed (16 rectangles, including squares as well as "flat," wider than taller, and "tall," taller than wider, rectangles); approximately half of the subjects disregarded the $90^{\circ}$ rotation that distinguished "flat" from "tall" rectangles. These subjects "folded" the physical design around the diagonals of the squares, thus reducing the set of 16 discriminable rectangles to 10 . Different psychophysical maps related the physical dimensions of the rectangles (e.g., height and width in centimeters) to the responses for each strategy group.

These findings clearly show that not all subjects process a physical stimulus design in the same way. They also suggest a categorization process in which subjects first create equivalence classes to simplify the task before concatenating the "dimensional" differences.

\section{Metrics and Transformations for Dissimilarity Judgments}

Since the first studies on "multidimensional psychophysics" (Richardson, 1938), dissimilarity ratings, $\delta(A, B)$, have been assumed to relate monotonically to the distances between the stimuli in the psychological space; that is, the combination operation is conceptualized as a distance or metric function that satisfies at least the three distance axioms (Equations 1-3) listed above.

Because in a direct dissimilarity rating task the response scale is determined by the experimenter (e.g., on a scale from 0 to 9 ), the responses necessarily satisfy the positivity condition of a distance function, Condition 1 . Sym metry, Condition 2, is usually assumed and/or forced by averaging or symmetrizing the matrix of dissimilarity responses. Hence, only the triangle inequality, Condition 3, is open to empirical disconfirmation. Schönemann et al. (1985) have defined data or metrics as "subadditive" if they satisfy the triangle inequality with strict inequality [i.e., $\delta(A, B)+\delta(B, C)>\delta(A, C)$ ]; as additive if strict equality holds [i.e., $\delta(A, B)+\delta(B, C)=\delta(A, C)$ ]; and as superadditive if the triangle inequality is violated [i.e., $\delta(A, B)+\delta(B, C)<\delta(A, C)]$.

The metric MDS literature (Torgerson, 1952, 1958) has emphasized the need to avoid superadditive observed dissimilarities, $\delta$; that is, the focus has been on violations of the triangle inequality. To restore the triangle inequality, it has been suggested to treat the $\delta$ s as "comparative distances" that relate to the "absolute distances," $d$, by an additive constant, $c: d(A, B)=\delta(A, B)+c$. Clearly, such an additive constant transformation can always restore the triangle inequality condition. For example, the triple $\delta(A, B)=3, \delta(B, C)=2$, and $\delta(A, C)=10$ violates the triangle inequality, but adding a value $c \geq 5$ restores the subadditivity. Later developments of nonmetric MDS (Kruskal, 1964; Shepard, 1962a, 1962b) allowed the transformation relating dissimilarities and distances to be any monotonic function that maximizes the fit of the specific (Minkowski) metric in hand. The joint fit of metric and ad hoc monotonic pretransformation, which characterizes most MDS algorithms, is not always recognized-even when its implications can be crucial to properly elucidate concepts such as integral versus separable stimuli (Garner, 1970; Garner \& Felfoldy, 1970).

However, most of the available empirical evidence on dissimilarity judgments of geometrical figures suggests that the triangle inequality condition is usually satisfied within sampling error. Furthermore, subadditivity is present not only across dimensions but also within a single dimension. Attneave (1950), recognizing this type of subadditivity, subtracted a constant to restore strict intradimensional additivity. Krantz and Tversky (1975), using an ordinal test for the "relative straightness" (i.e., additivity), of the dimensions, reported differential "additivity" for their dimensions. Schönemann et al. (1985) and Schönemann and Lazarte (1987), using the direct, un- 
transformed observed responses, reported consistent subadditivity in their data for rectangles along any coordinate system $(H / W$ or $A / S)$, within the same dimension and between dimensions. Borg and Leutner (1983) reported similar results.

If the dissimilarity ratings satisfy the positivity and symmetry conditions of a metric due to the constraints of the rating task, and if dissimilarities also satisfy the triangular inequality, they can always be modeled as distances. However, because the observed direct dissimilarities are consistently segmentally subadditive along any possible judgment dimension, they cannot be modeled as Minkowski metrics, because these metrics assume intradimensional additivity. As a possible alternative subadditive metric, Schönemann $(1982,1983)$ has postulated a "metric for bounded responses"' (MBR).

\section{Metric for Bounded Responses (MBR)}

Psychologically, this metric can be derived from two assumptions: (1) Individuals facing a direct rating dissimilarity task perform simple cognitive operations such as concatenating the differences of the stimulus coordinates projected on the axes, and (2) subjects have to contract their responses to produce a numerical answer within the upper bound imposed by the experimenter's response scale. The first assumption can be considered as a city-block metric requirement. The second imposes a transformation on the data that accounts for the subadditivity.

The MBR for stimuli projecting on two dimensions can be expressed as:

$$
d^{*}(A, B)=(a+b) /(1+a b) \quad 0 \leq a, b, d^{*}(A, B)<1,
$$

where $d^{*}(A, B)$ is the observed relative dissimilarity between the stimuli $A$ and $B$ (relative to the upper bound value in the response scale), and $a$ and $b$ are the segments projected by the difference of the stimuli coordinates on the underlying subjective dimensions. Schönemann (1983) has shown that Equation 6 is indeed a metric that emulates the city-block metric at the lower end of the $[0,1)$ interval, the dominance or supremum metric at the upper end of $[0,1)$, and the Euclidean distance in the middle range.

Equation 6 can be expressed in terms of the hyperbolic tangent transformation as:

$$
d^{*}(A, B)=\tanh (u+v)=(a+b) /(1+a b) \quad 0 \leq u, v<\infty,
$$

where $a=\tanh (u), b=\tanh (v), 0 \leq a, b, d^{*}(A, B)<1$. Therefore, the inverse hyperbolic tangent transformation of the observed relative dissimilarities,

$$
\tanh ^{-1}\left[d^{*}(A, B)\right]=u+v=\tanh ^{-1}(a)+\tanh ^{-1}(b),
$$

leads back to the city-block metric on the unbounded segmental projections $u, v$.

Schönemann et al. (1985) and Schönemann and Lazarte (1987), after comparing different subadditive concatenations, found that within subjects, the MBR with a slightly reduced upper bound was optimal in restoring additivity among collinear pairs. At the group level, the simple MBR produced the best MDS solution fit. This confirmed the prediction that the MDS solution of the data using the city-block metric with the hyperbolic tangent transformation markedly improved the fit.

\section{Psychophysical Maps with MBR Pretransformation}

In Schönemann and Lazarte (1987), the traditional MDS perspective, which basically involved the use of subjective coordinates to explain the dissimilarity data, was replaced with a psychophysical approach, relating the observed dissimilarities directly to the physical dimensions of the stimuli. The MDS solutions, using MBR, suggested the following representation for the heightwidth (HW) groups:

$$
\tanh ^{-1}\left[d_{i j}^{*}\right]=h\left[H_{i}, H_{j}, W_{i}, W_{j}\right],
$$

where $d_{i j}^{*}$ is the observed relative dissimilarity between stimuli $i$ and $j, d_{i j}^{*}=d_{i j} / k, d_{i j}=$ observed dissimilarity, $k=$ maximum possible value in the response scale, and $H_{i}, H_{j}, W_{i}$, and $W_{j}$ are the coordinates of the stimuli on physical height and width.

The function $h$ was derived from the analysis of the responses to the squares included in the physical design (see Figure 1), and turned out to be linear in the arguments, thus giving the following four-parameter model for the HW group:

$$
\tanh ^{-1}\left[d_{i j}^{*}\right]=\beta_{1} W_{L}-\beta_{2} W_{S}+\beta_{3} H_{L}-\beta_{4} H_{S},
$$

where $W_{L}=\operatorname{Max}\left\{W_{i}, W_{j}\right\}, W_{S}=\operatorname{Min}\left\{W_{i}, W_{j}\right\}, H_{L}=\operatorname{Max}\left\{H_{i}, H_{j}\right\}, H_{S}=\operatorname{Min}\left\{H_{i}, H_{j}\right\}$. 
For the area-shape (AS) groups, two derived indices were used that followed the same idea of simple linear combinations of height and width. Area was coded as $A=(H+W) / 2$ and shape was defined as "eccentricity," $X$, or departure from squareness-that is, $X=|H-W| / 2$. For the AS groups, the four-parameter model then became:

$$
\tanh ^{-1}\left[d_{i j}^{*}\right]=\tau_{1} X_{L}-\tau_{2} X_{S}+\tau_{3} A_{L}-\tau_{4} A_{S},
$$

where $X_{L}=\operatorname{Max}\left\{X_{i}, X_{j}\right\}, X_{S}=\operatorname{Min}\left\{X_{i}, X_{j}\right\}, A_{L}=\operatorname{Max}\left\{A_{i}, A_{j}\right\}, A_{S}=\operatorname{Min}\left\{A_{i}, A_{j}\right\}$. For the subjects who used only eccentricity in rating dissimilarities, Group $X$, Model 11 fit best with the weights for the area components close to zero.

We obtained excellent fits for Models 10 and 11, and also for approximations that involved fewer parameters (see Table 1). We concluded that the $\tanh ^{-1}$ transformation helped to improve the fit of the psychophysical maps.

In summary, three general conclusions can be obtained from our previous research on dissimilarity rating of rectangles: (1) The dimensional orientation is not necessarily the same for all subjects; different subjects use different strategies. (2) Regardless of strategy, the combination rule used by the subjects is segmentally subadditive, producing ratings that satisfy the triangular inequality and therefore are compatible with any subadditive metric, but not with Minkowski metrics. (3) With the help of the MBR transformation, dissimilarity ratings can be related to a linear, additive combination of the rectangles' physical dimensions.

The present study was intended, in part, as a further test of Models 10 and 11 when a smaller number of stimuli (see Figure 1) provides a sharper split between alternative strategies. Within each strategy, we expected to find subadditive ratings and to be able to model them as a concatenation of physical dimensions rendered additive by the MBR transformation.

On the other hand, Equations 10 and 11 can be considered as a subadditive metric function defined now over physical dimensions. Because in our previous studies the dissimilarity rating data were subadditive, a further test of the equations will consist in contrasting their fits against the fits for the untransformed observed relative dissimilarity ratings $d_{i j}^{*}$

$$
\begin{aligned}
& d_{i j}^{*}=\beta_{1} W_{L}-\beta_{2} W_{S}+\beta_{3} H_{L}-\beta_{4} H_{S}, \\
& d_{i j}^{*}=\tau_{1} X_{L}-\tau_{2} X_{S}+\tau_{3} A_{L}-\tau_{4} A_{S} .
\end{aligned}
$$

On the basis of our previous findings, we expected the fits for Equations 10 and 11 to be substantially better than those for Equations 12 and 13.

\section{METHOD}

\section{Subjects}

The subjects were 60 unpaid undergraduates of both genders, fulfilling their research participation requirement for an introductory course in psychology at Purdue University.

\section{Apparatus and Stimuli}

Nine solid-green rectangles, against a dark background, were presented in pairs to the subjects, on a Zenith ZVM1240 monochrome monitor. The presentation of instructions and stimuli and the recording of responses were controlled by a BASIC program run by a Zenith ZDH-1111-B0 microcomputer. The stimulus design was a $3 \times 3$, completely crossed in a height and width coordinate system (Figure 1). The stimuli were a subset of the stimulus design used previously by Schönemann and Lazarte (1987). The values for both width and height were 2,4 , and $6 \mathrm{~cm}$. On each presentation, a pair of rectangles appeared side by side on the screen, and the right-left screen position was determined at random for each pair. The presentation sequence was random for half of the subjects and fixed for the other half of the subjects. The 36 different pairs were presented four times each. Pairs with identical rectangles were omitted. The subjects responded by pressing one of the numeric keys; the other keys were locked.

\section{Procedure}

Subjects were tested individually in groups of up to 6 individuals. Each subject was asked to press any key to start the presentation of the instructions and practice trials. The subjects rated the dissimilarity of each pair of rectangles on a scale from 0 (identical) to 9 (maximally different). To familiarize the subjects with the stimuli, each rectangle was displayed individually on the screen for $5 \mathrm{sec}$ prior to the practice trials. Before the actual experimental session, the subjects practiced the response procedure, using at least 10 practice trials. These trials were a random sample of real experimental trials.

On the actual experimental trials, pairs of rectangles were displayed. When the subjects pressed a valid numerical key, the screen was cleared and a new pair appeared immediately, allowing the subjects to work at their own speeds. The responses and the time required for each rating were recorded and stored following each trial. 
Table 1

Fit Indices for Psychophysical Maps of Rectangles Using tanh $^{-1}$ Transformation*

\begin{tabular}{|c|c|c|c|c|c|c|}
\hline \multirow[b]{3}{*}{ Group } & \multicolumn{6}{|c|}{ Psychophysical Maps } \\
\hline & \multicolumn{2}{|c|}{$\begin{array}{c}\text { Four } \\
\text { Parameters }\end{array}$} & \multicolumn{2}{|c|}{$\begin{array}{c}\text { Unweighted } \\
\text { One Parameter }\end{array}$} & \multicolumn{2}{|c|}{$\begin{array}{c}\text { Weighted } \\
\text { One Parameter }\end{array}$} \\
\hline & $\mathrm{eta}^{2}$ & $r$ & eta $^{2}$ & $r$ & eta $^{2}$ & $r$ \\
\hline HW1 & .97 & .92 & .79 & .78 & .89 & .73 \\
\hline HW2 & .98 & .92 & .52 & .90 & .98 & .90 \\
\hline $\mathrm{AX} 1$ & .95 & .82 & .50 & .83 & .83 & .79 \\
\hline $\mathrm{AX} 2$ & .95 & .84 & .45 & .81 & .64 & .77 \\
\hline $\mathbf{x}$ & .95 & .78 & .56 & .60 & .86 & .66 \\
\hline Mean & .96 & .86 & .56 & .78 & .84 & .77 \\
\hline
\end{tabular}

Note-Eta ${ }^{2}$ is given by dividing the (uncorrected) model sum of squares by the (uncorrected) total sum of squares. The values of $r$ correspond to the correlation between the predictions from the (no intercept) model and the observed values. HW1 and HW2 refer to height and width strategy groups. AX1 and AX2 refer to area and eccentricity strategy groups. $\mathrm{X}$ is the only eccentricity strategy group. *Adapted from Table 5 of Schönemann and Lazarte (1987).

\section{RESULTS}

\section{Individual Differences}

As in our previous study (see Schönemann \& Lazarte, 1987, and also Table 1 here), we classified subjects on the basis of their responses to the stimulus pairs on the two main diagonals of the stimuli design: (1) the observed dissimilarity between Stimuli 3 and $7, d(3,7)$-the maximal shape difference-and (2) the observed dissimilarity $d(1,9)$-the maximal size difference. The resulting cross-classification table is presented in Table 2.

Fifty-three subjects assigned a value of three or less to the shape-diagonal dissimilarity, while the remaining 7 subjects gave it a value of five or more, thus providing

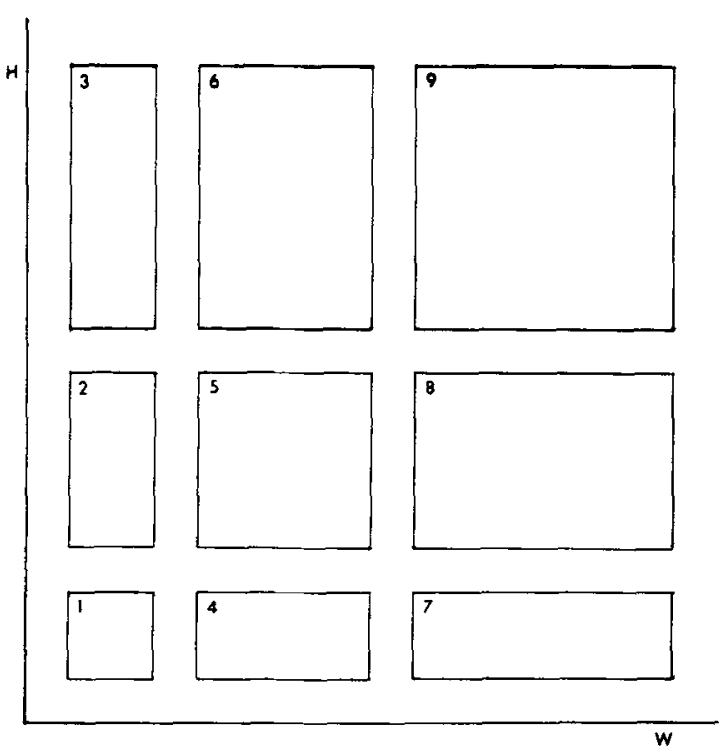

Figure 1. Stimulus design, along height and width, and numerical labels of rectangles used in text, tables, and figures. The diagonal through Stimuli 1, 5, and 9 is the area diagonal, and the diagonal through Stimuli 3, 5, and 7 is the shape diagonal. Both height and width have values of 2,4 , and $6 \mathrm{~cm}$. a natural cutting point on the marginal frequency distribution of the shape diagonal. Although the area diagonal did not show such a clear division, we set the cutting line between ratings of two and three. This classification generated three groups: an AX (area and eccentricity) group (45 subjects) judged the shape diagonal to have a smaller average dissimilarity than the area diagonal; an HW (height and width) group (6 subjects) judged both diagonals as relatively large; and the $X$ (eccentricity) group ( 9 subjects) judged neither shape nor size with large dissimilarities.

The AX group, containing the majority of the subjects, assigned a very small or even zero value to $d(3,7)$, which means that they treated rectangles with the same eccentricity, differing only by a $90^{\circ}$ rotation, as equivalent. Thus, this group collapsed the design along the area diagonal, therefore reducing the 36 different comparisons (pairs) to only 15 . In order to cross-validate the numerical estimates presented later, and to obtain groups of approximately the same sizes, the AX group was randomly subdivided into five subgroups of 9 subjects each.

The 9 subjects in the $X$ group not only collapsed across the shape diagonal, but also gave small dissimilarity ratings to the area diagonal. This group simplified the task to a choice between squares and rectangles. The remaining 6 subjects in the HW group used height and width almost equally.

In general, the classification of the subjects by $\mathrm{HW}$ and AX strategies is similar to previous findings (Schönemann \& Lazarte, 1987). However, the percentages of subjects in each group are different: with the present stimulus design, the AX strategy is the one most often used. The average relative dissimilarities (i.e., average response divided by 9) for the three groups are listed in Table 3.

\section{The Metric Problem: Direct Analysis of Composition Rules}

As in Schönemann et al. (1985), the fit for the cityblock, supremum, Euclidean, average, and average power metrics, and different versions of MBR metrics were compared. The different composition rules, $c=g(a, b)$, were fit to observed relative dissimilarities, $a, b$, and $c$, for triples of pairs along the height-width and area-eccentricity dimensions. To illustrate this in an area and eccentricity coordinate system, for the triple of pairs defined by Stimuli 1, 5, and 9 , the city-block metric predicts that $g(a, b)=c=a+b$; thus $d^{*}(1,9)=d^{*}(1,5)+d^{*}(5,9)$, where $d^{*}(.,$.$) is the observed relative dissimilarity between$ two stimuli. The fit of each composition rule was evaluated in terms of the residuals, $g(a, b)-c$, where $g$ is the prediction and $c$ the observed value of the particular composition rule. The mean of the residuals indicates the bias of the composition rule, and their (uncorrected) sums of squares the actual fit. The following composition rules were investigated:

$\begin{array}{ll}\text { CB } & :=\text { City-block metric: } c=a+b . \\ \text { SUP } & :=\text { Supremum metric: } c=\max \{a, b\} . \\ \text { EUC } & :=\text { Euclidean metric: } c=\left(a^{2}+b^{2}\right)^{1 / 2} \\ \text { MBR(L) } & :=\text { Metric for bounded responses: }\end{array}$


Table 2

Scatterplot of Area, $d(1,9)$, and Shape, $d(3,7)$, Diagonals Used to Define Strategy Groups

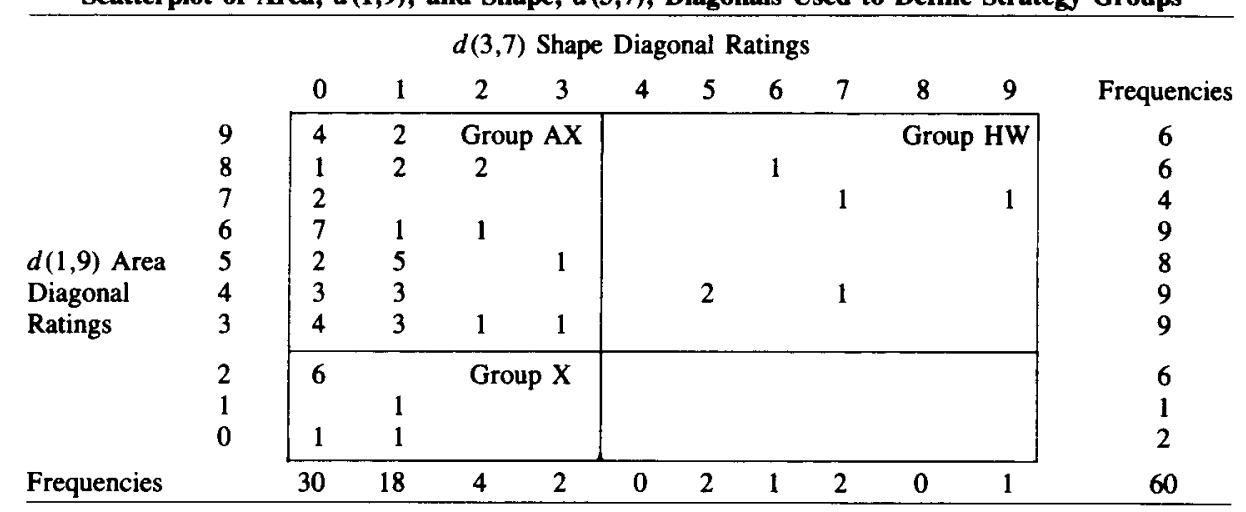

$c=(a+b) /(1+a b)$, where $L=9$ is the upper bound of the response scale used to compute the relative dissimilarities, $d / L$.

MBR(sL) := Same as MBR(L), but with a reduced upper bound $s L$, with $s=.8$.

$\operatorname{MBR}(\mathrm{MX}):=$ Same as $\operatorname{MBR}(\mathrm{L})$, where the upper bound, $L$, is replaced by the maximal response of the subject.

AVER := Average: $c=(a+b) / 2$.

AVPW $\quad:=$ Average Power: $c=\left(a^{1 / 2}+b^{1 / 2}\right) / 2$.

For each composition rule, the residual means and residual sum of squares for collinear triples along height and width dimensions, and along the area and shape diagonals, are shown in Tables $4 \mathrm{~A}$ and $4 \mathrm{~B}$ for each group of subjects.

Table 3

Relative Average Dissimilarities Between Pairs of Stimuli for the Three Major Classification Groups

AX Group $(\mathrm{N}=45)$ above diagonal

HW Group $(\mathrm{N}=6)$ below diagonal

\begin{tabular}{|c|c|c|c|c|c|c|c|c|c|}
\hline & \multicolumn{9}{|c|}{ Stimulus Number } \\
\hline & 1 & 2 & 3 & 4 & 5 & 6 & 7 & 8 & 9 \\
\hline 1 & & 0.52 & 0.67 & 0.48 & 0.45 & 0.81 & 0.63 & 0.81 & 0.61 \\
\hline 2 & 0.52 & & 0.39 & 0.09 & 0.54 & 0.56 & 0.33 & 0.68 & 0.85 \\
\hline 3 & 0.66 & 0.39 & & 0.36 & 0.77 & 0.49 & 0.08 & 0.61 & 0.71 \\
\hline 4 & 0.47 & 0.70 & 0.69 & & 0.64 & 0.70 & 0.34 & 0.56 & 0.87 \\
\hline 5 & 0.42 & 0.51 & 0.81 & 0.63 & & 0.54 & 0.76 & 0.44 & 0.41 \\
\hline 6 & 0.86 & 0.54 & 0.43 & 0.68 & 0.48 & & 0.57 & 0.10 & 0.42 \\
\hline 7 & 0.59 & 0.65 & 0.72 & 0.35 & 0.84 & 0.80 & & 0.56 & 0.81 \\
\hline 8 & 0.86 & 0.66 & 0.81 & 0.60 & 0.43 & 0.65 & 0.55 & & 0.54 \\
\hline $\begin{array}{l}9 \\
\text {. }\end{array}$ & 0.64 & 0.88 & 0.62 & 0.87 & 0.39 & 0.32 & 0.66 & 0.46 & \\
\hline \multicolumn{10}{|c|}{ X Group $(\mathrm{N}=9)$} \\
\hline & \multicolumn{9}{|c|}{ Stimulus Number } \\
\hline & 1 & 2 & 3 & 4 & 5 & 6 & 7 & 8 & 9 \\
\hline | & 0.00 & 0.59 & 0.67 & 0.58 & 0.20 & 0.68 & 0.67 & 0.75 & 0.20 \\
\hline 2 & & 0.00 & 0.28 & 0.10 & 0.61 & 0.32 & 0.22 & 0.56 & 0.70 \\
\hline 3 & & & 0.00 & 0.27 & 0.74 & 0.44 & 0.09 & 0.53 & 0.70 \\
\hline 4 & & & & 0.00 & 0.66 & 0.47 & 0.31 & 0.33 & 0.69 \\
\hline 5 & & & & & 0.00 & 0.50 & 0.73 & 0.58 & 0.20 \\
\hline 6 & & & & & & 0.00 & 0.38 & 0.12 & 0.57 \\
\hline . & & & & & & & 0.00 & 0.47 & 0.73 \\
\hline & & & & & & & & 0.00 & 0.60 \\
\hline & & & & & & & & & 0.00 \\
\hline
\end{tabular}

For the relative dissimilarity responses, which range between $(0,1)$, the predictions, $g(a, b)$, based on the average (AVR), supremum (SUP), Euclidean (EUC), and cityblock (CB) metric follow the ordering AVR $\leq$ SUP $\leq$ EUC $\leq \mathrm{CB}$ for any $a, b \in(0,1)$, thus:

$$
(a+b) / 2 \leq \max (a, b) \leq\left(a^{2}+b^{2}\right)^{1 / 2} \leq a+b .
$$

As in our earlier studies, the city-block metric produced a substantial positive bias across all groups and comparisons, which means that the data were again consistently subadditive. On the other hand, the biases for the averaging function, and to a lesser degree the biases for the supremum function, were consistently negative, which means that these two functions were too subadditive for the data. The bias for the MBR functions, the Euclidean, and the average power functions fell mostly between -.1 and +.1 . The various versions of the MBR were least biased and among the more effective in restoring additivity. Along the shape diagonal, all the biases were large and positive as a result of the near zero rating that most of the subjects assigned to the pair $(3,7)$.

A relative measure of fit for each composition rule is given by the ratio of its (uncorrected) residual sum of squares (SSQ) over the residual sum of squares left by the city-block metric (see Table 5). The MBR metric with a reduced upper bound $[M(s 9)$, with $s=.8]$ tends to produce a smaller SSQ than any of the other metrics, including the direct $\mathrm{MBR}(\mathrm{L})$.

Overall, the results reported so far confirmed previous findings. The pervasive subadditivity in dissimilarity data and the effectiveness of the inverse hyperbolic tangent transformation in removing the subadditivity at the withinsubjects level were again demonstrated.

\section{Psychophysical Maps: MBR Transformation versus Direct Responses}

The least squares fits for the four-parameter model using the $\tanh ^{-1}$ pretransformation, Equations 10 and 11 , and for the four-parameter model without transformation, Equations 12 and 13, are compared in Table 6.

Contrary to what was expected on the basis of the earlier studies, the values of eta $^{2}$ using the MBR transformation were actually slightly smaller than the values obtained 
without the transformation, although the correlations, $r$, between observed and predicted values were slightly higher under the $\tanh ^{-1}$ transformation. However, the conclusion about which model fit better was unaffected by the transformation. The responses of the $\mathrm{AX}$ groups were described better by the AX model (an average eta ${ }^{2}$ of .95 and $r$ of .83 ), while the HW model fit the responses of the HW group better (eta $\left.{ }^{2}=.95, r=.69\right)$. The differential fits confirmed the classification of the subjects into different strategy groups on the basis of their responses to only two of the rectangle pairs as shown in Table 2. The AX Model four-parameter maps for the five AX subgroups and the $X$ group were:

$$
\begin{aligned}
& \text { AX1 group: } \quad d^{*}=.17 X_{L}-.21 X_{S}+.17 A_{L}-.11 A_{S} \text {, } \\
& \tanh ^{-1}\left(d^{*}\right)=.24 X_{L}-.31 X_{S}+.23 A_{L}-.18 A_{S} \text {. } \\
& \text { AX2 group: } \quad d^{*}=.15 X_{L}-.18 X_{S}+.17 A_{L}-.12 A_{S} \text {, } \\
& \tanh ^{-1}\left(d^{*}\right)=.19 X_{L}-.24 X_{S}+.22 A_{L}-.17 A_{S} \text {. } \\
& \text { AX3 group: } \quad d^{*}=.17 X_{L}-.18 X_{S}+.17 A_{L}-.13 A_{S} \text {, } \\
& \tanh ^{-1}\left(d^{*}\right)=.24 X_{L}-.27 X_{S}+.23 A_{L}-.19 A_{S} . \\
& \text { AX4 group: } \quad d^{*}=.17 X_{L}-.16 X_{S}+.16 A_{L}-.12 A_{S} \text {, } \\
& \tanh ^{-1}\left(d^{*}\right)=.23 X_{L}-.24 X_{S}+.21 A_{L}-.17 A_{S} \text {. } \\
& \text { AX5 group: } \quad d^{*}=.19 X_{L}-.18 X_{S}+.17 A_{L}-.11 A_{S} \text {, } \\
& \tanh ^{-1}\left(d^{*}\right)=.27 X_{L}-.28 X_{S}+.23 A_{L}-.17 A_{s} . \\
& \text { All AX groups: } \quad d^{*}=.17 X_{L}-.18 X_{S}+.16 A_{L}-.12 A s \text {, } \\
& \tanh ^{-1}\left(d^{*}\right)=.23 X_{L}-.26 X_{S}+.22 A_{L}-.18 A_{S} . \\
& \mathrm{X} \text { group: } \quad d^{*}=.24 X_{L}-.38 X_{S}+.08 A_{L}-.02 A_{S} \text {, } \\
& \tanh ^{-1}\left(d^{*}\right)=.31 X_{L}-.51 X_{S}+.09 A_{L}-.03 A_{S} \text {. }
\end{aligned}
$$

In Schönemann and Lazarte (1987), we found that the weights of the larger coordinates (in centimeters) on each physical dimension were consistently larger, in absolute value, than the weights of the smaller coordinates, which was interpreted as a "saliency" effect of the larger coordinate. After taking this ordering of the weights into account, Schönemann and Lazarte (1987) were able to approximate the four-parameter maps with models involving fewer parameters.

In Equations $14 \mathrm{~A}-14 \mathrm{~N}$, the area weights followed the expected ordering, $A_{L}>A s$. However, in three of the $\mathrm{AX}$ subgroups (Equations $14 \mathrm{~A}-14 \mathrm{~F}$ ), and in the $\mathrm{X}$ group (Equations $14 \mathrm{M}, 14 \mathrm{~N}$ ), the weights for the smaller eccentricity coordinate, $X_{S}$, were larger than the weights for $X_{L}$. These order reversals held up with and without the MBR transformation.

The map for the HW group was:

$$
\text { HW group: } \begin{aligned}
d^{*} & =.09 W_{L}-.05 W_{S}+.10 H_{L}-.07 H_{S}, \\
\tanh ^{-1}\left(d^{*}\right) & =.13 W_{L}-.09 W_{S}+.15 H_{L}-.13 H_{S} .
\end{aligned}
$$

In this case, the weights for the larger coordinates, $W_{L}$ and $H_{L}$, were consistently larger than those for the smaller coordinates.

The results of fitting these psychophysical maps raised two new questions: (1) Why did the MBR transformation not substantially improve the fit of the maps; and (2) why did the ordering of the weights not consistently agree with previous findings that $X_{L}>X_{S}$ ?

To answer the first question, we recall that we used the MBR transformation to restore additivity in the concatenations of the physical coordinates, because the within-subjects analysis revealed pervasive subadditivity. However, as it turns out, a four-parameter linear model by itself predicts a subadditive concatenation, so that the effect of the $\tanh ^{-1}$ correction is reduced or even neutralized. The extent of the subadditivity predicted by the four-parameter model depends on the values of the weights in the equation. As will also be shown below, expressions analogous to Equations 12 and 13 imply subadditive predictions when the weights for the larger coordinates are larger than the weights for the smaller coordinates. Alternatively, one may view the four-parameter model as implying a subadditive saliency metric that emphasizes the larger coordinate. In contrast to the MBR, this saliency metric is not invariant under translation. 
Saliency Metric: A Subadditive Metric with No Translational Invariance

A general expression for the four-parameter model is given by

$$
d_{i j}=\left(\beta_{1} X_{L}-\beta_{2} X_{S}\right)+\left(\beta_{3} Y_{L}-\beta_{4} Y_{S}\right) \text {, }
$$

where the stimuli $i$ and $j$ are located on a two-dimensional coordinate system with coordinates $\left(X_{i}, Y_{i}\right)$ and $\left(X_{j}, Y_{j}\right)$, and $X_{L}=\operatorname{Max}\left\{X_{i}, X_{j}\right\}, X_{S}=\operatorname{Min}\left\{X_{i}, X_{j}\right\}, Y_{L}=\operatorname{Max}\left\{Y_{i}, Y_{j}\right\}, Y_{S}=\operatorname{Min}\left\{Y_{i}, Y_{j}\right\}$. Furthermore, $X_{L}, X_{S}, Y_{L}, Y_{S}>0$, and $\beta_{1}, \beta_{2}, \beta_{3}, \beta_{4} \geq 0$.

Equation 16 can be rearranged in terms of the intradimensional segmental differences $a=$ $\left(X_{L}-X_{S}\right), b=\left(Y_{L}-Y_{S}\right)$ in two ways (see Figure 2):

$$
d_{i j}=\beta_{1} a+\beta_{3} b+\left(\beta_{1}-\beta_{2}\right) X_{S}+\left(\beta_{3}-\beta_{4}\right) Y_{S}
$$

or

$$
d_{i j}=\beta_{2} a+\beta_{4} b+\left(\beta_{1}-\beta_{2}\right) X_{L}+\left(\beta_{3}-\beta_{4}\right) Y_{L} .
$$

On averaging both expressions, one obtains

$$
d_{i j}=\left[\left(\beta_{1}+\beta_{2}\right) / 2\right] a+\left[\left(\beta_{3}+\beta_{4}\right) / 2\right] b+\left(\beta_{1}-\beta_{2}\right) \bar{X}_{i j}+\left(\beta_{3}-\beta_{4}\right) Y_{i j},
$$

where $\bar{X}_{i j}=\left(X_{L}+X_{S}\right) / 2$ and $Y_{i j}=\left(Y_{L}+Y_{S}\right) / 2$ are the mean coordinates, so that $\left(X_{i j}, Y_{i j}\right)$ is the centroid (point of gravity) of the line segment connecting Stimuli $i$ and $j$.

In Equation 17A, $\beta_{1}$ and $\beta_{3}$ can be considered scaling parameters, while $\left(\beta_{1}-\beta_{2}\right)$ and $\left(\beta_{3}-\beta_{4}\right)$ determine the difference in the weighing for the large and small values in the respective dimension, a saliency parameter that indicates by how much the larger projection is weighed over the smaller one. Equations 17A, 17B, and 18A show that the four-parameter model can be expressed as a city-block concatenation of the projections $a$ and $b$, plus two additional terms that become zero only if $\beta_{1}=\beta_{2}$ and $\beta_{3}=\beta_{4}$-that is, if there is no saliency effect. Note that these additional terms destroy translational invariance, making the distance depend on the specific location of the two stimuli in the space.

J. C. Dunn, in his review of the present paper, suggested an alternative way of expressing the saliency metric:

$$
d_{i j}=\alpha_{1}\left(\beta_{X} X_{L}-X_{S}\right)+\alpha_{2}\left(\beta_{Y} Y_{L}-Y_{S}\right),
$$

where $\alpha_{1} \geq 0$ and $\alpha_{2} \geq 0$ are interpretable as the relative saliencies of the $X$ and $Y$ dimensions, and $\beta_{X} \geq 1$ and $\beta_{Y} \geq 1$ represent the saliency of the largest coordinate on each dimension. Although Equation 18B does not immediately show the lack of translational invariance, it can help to simplify the proofs of the metric properties.

Proposition: Equation 16 is a subadditive metric that satisfies the properties of positivity, minimality for equal stimuli, symmetry, and triangular inequality.

Proof:

\section{Positivity}

Let $X_{L}, X_{S}, Y_{L}, Y_{S} \geq 0$, and $\beta_{1}, \beta_{2}, \beta_{3}, \beta_{4}>0$. A plausible requirement would be to restrict the weighted projections $\left(\beta_{1} X_{L}-\beta_{2} X_{S}\right)$ and $\left(\beta_{3} Y_{L}-\beta_{4} Y_{S}\right)$ to be positive. For $X_{L}, X_{S}$, then we have that:

$$
\begin{aligned}
\left(\beta_{1} X_{L}-\beta_{2} X_{S}\right)= & \beta_{1}\left(X_{L}-X_{S}\right)+\left(\beta_{1}-\beta_{2}\right) X_{S} \geq 0 \\
& \left(X_{L}-X_{S}\right) \geq-\left[\left(\beta_{1}-\beta_{2}\right) / \beta_{1}\right] X_{S} \\
& \left(X_{L}-X_{S}\right) \geq\left(\beta_{2} / \beta_{1}-1\right) X_{S} .
\end{aligned}
$$

Since $\left(X_{L}-X_{S}\right) \geq 0$ by definition, Equation 20 expresses the actual lower bound in terms of the beta parameters. If $\beta_{1}>\beta_{2}$, then the right-hand side in Equation 20 is negative and therefore Equations 19 and 20 are satisfied for any $X_{S}$ and $X_{L}$. If $\beta_{1}<\beta_{2}$, then the right-hand side in Equation 20 is a positive, nontrivial, lower bound and Equation 19 is satisfied only if $X_{L} \geq\left(\beta_{2} / \beta_{1}\right) X_{S}$. (Note that in this case, for $X_{L}=X_{S}$, but not zero, the projection will always be negative.) In general, then, we require that

$$
\beta_{1} \geq \beta_{2} \text { and } \beta_{3} \geq \beta_{4},
$$

in order for Equation 16 to be always positive regardless of specific values for the $X$ and $Y$ coor- 
dinate points. This justifies the requirement of $\beta_{X} \geq 1$ and $\beta_{Y} \geq 1$ in Dunn's equation, and makes evident that Equation $18 \mathrm{~B}$ is always positive. Thus, if $\beta_{1} \geq \beta_{2}$ and $\beta_{3} \geq \beta_{4}$, then $d_{i j} \geq 0$ for any $i, j$.

\section{Minimality}

Let $i$ be any stimulus with coordinates $\left(X_{i}, Y_{i}\right)$. For $d_{i i}$, then $X_{L}=X_{S}=X_{i}$ and $Y_{L}=Y_{S}=Y_{i}$; therefore, $d_{i i}=\left(\beta_{1}-\beta_{2}\right) X_{i}+\left(\beta_{3}-\beta_{4}\right) Y_{i}$, which in general is not zero. Let $j$ be another stimulus with $\left(X_{j}, Y_{j}\right)$, such that $X_{i} \neq X_{j}$ and/or $Y_{i} \neq Y_{j}$. Let $X_{j}>X_{i}$ and $Y_{j}<Y_{i}$; therefore, $X_{L}=X_{j}$ and $Y_{L}=Y_{i}$, and replacing in Equation 16

$$
\begin{aligned}
d_{i j} & =\left(\beta_{1} X_{j}-\beta_{2} X_{i}\right)+\left(\beta_{3} Y_{i}-\beta_{4} Y_{j}\right), \\
d_{i j} & =\left(\beta_{1}-\beta_{2}\right) X_{i}+\beta_{1}\left(X_{j}-X_{i}\right)+\left(\beta_{3}-\beta_{4}\right) Y_{i}+\beta_{4}\left(Y_{i}-Y_{j}\right), \\
d_{i j} & =\underbrace{\left(\beta_{1}-\beta_{2}\right) X_{i}+\left(\beta_{3}-\beta_{4}\right) Y_{i}+\beta_{1}\left(X_{j}-X_{i}\right)+\beta_{4}\left(Y_{i}-Y_{j}\right),}_{d_{i i}},
\end{aligned}
$$

where $K>0$, since we assumed $X_{j}>X_{i}$ and $Y_{j}<Y_{i}$. Similar results are obtained for any assignment of $X_{L}$ and $Y_{L}$; therefore, $d_{i j}>d_{i i}$ for all $i j$. Using Equation 18B, it is evident that $d_{i j}$ is minimum when $i=j$.

\section{Symmetry}

In Equation 16, $d_{i j}$ is defined in terms of $X_{L}=\operatorname{Max}\left\{X_{i}, X_{j}\right\}, X_{S}=\operatorname{Min}\left\{X_{i}, X_{j}\right\}, Y_{L}=\operatorname{Max}\left\{Y_{i}, Y_{j}\right\}$, $Y_{S}=\operatorname{Min}\left\{Y_{i}, Y_{j}\right\}$, and since the Max and Min functions are symmetric, then $d_{i j}=d_{j i}$.

\section{Triangle inequality}

Let $A, B$, and $C$ represent 3 stimuli as in Figure 3, and the equivalent form (Equation 17A) $d_{i j}=$ $\beta_{1} a+\beta_{3} b+\left(\beta_{1}-\beta_{2}\right) X_{S}+\left(\beta_{3}-\beta_{4}\right) Y_{S}$. For the distance between $A$ and $B$, the smallest coordinates are represented by $X_{S 1}$ and $Y_{S 1}$, which are also the smallest coordinates for the distance between $A$ and $C$. The smallest coordinates for $B$ and $C$ are denoted by $X_{S 2}$ and $Y_{S 2}$.

Denote $\left(\beta_{1}-\beta_{2}\right) X_{S}+\left(\beta_{3}-\beta_{4}\right) Y_{S}$ by $g\left(X_{S}, Y_{S}\right)$, where, from the positivity restrictions (Equation 21), $g\left(X_{S}, Y_{S}\right) \geq 0$. Let $a+p=r$ and $b+q=s$, so:

$$
\begin{aligned}
& d_{A B}=\beta_{1} a+\beta_{3} b+g\left(X_{S 1}, Y_{S 1}\right), \\
& d_{B C}=\beta_{1} p+\beta_{3} q+g\left(X_{S 2}, Y_{S 2}\right), \\
& d_{A C}=\beta_{1} r+\beta_{3} s+g\left(X_{S 1}, Y_{S 1}\right),
\end{aligned}
$$

then:

$$
d_{A B}+d_{B C}=\underbrace{\beta_{1} r+\beta_{3} s+g\left(X_{S 1}, Y_{S 1}\right)}_{\mathrm{d}_{A C}}+\underbrace{g\left(X_{S 2}, Y_{S 2}\right)}_{\geq 0} .
$$

Thus, $d_{A B}+d_{B C} \geq d_{A C}$. Considering the positivity condition, Equation 16 violates the triangle inequality when both restrictions $\beta_{1} \geq \beta_{2}$ and $\beta_{3} \geq \beta_{4}$ are not fulfilled. A similar conclusion is obtained when the point $B$ in Figure 3 has a vertical coordinate higher than point $C$.

\section{Intrasegmental subadditivity}

Equation 16 is also intrasegmentally subadditive. Consider $A, B$, and $C$ that are collinear along $Y$-that is, $Y_{S 1}=Y_{S 2}=Y$-and $b=q=s=0$ (in Figure 3); then Equations 23 reduce to

$$
\begin{aligned}
& d_{A B}=\beta_{1} a+g\left(X_{S 1}, Y\right), \\
& d_{B C}=\beta_{1} p+g\left(X_{S 2}, Y\right), \\
& d_{A C}=\beta_{1} r+g\left(X_{S 1}, Y\right) .
\end{aligned}
$$

Therefore,

$$
d_{A B}+d_{B C}=\underbrace{\beta_{1} r+g\left(X_{S 1}, Y\right)}_{d_{A C}}+\underbrace{g\left(X_{S 2}, Y\right)}_{\geq 0} .
$$

Segmental additivity only occurs when $g\left(X_{S 2}, Y\right)=0$-that is, when $\left(\beta_{1}-\beta_{2}\right) X_{S}+\left(\beta_{3}-\beta_{4}\right) Y_{S}$ $=0$, or when $\beta_{1}=\beta_{2}$ and $\beta_{3}=\beta_{4}$, in which case Equation 16 reduces to a city-block metric with scaling parameters $\beta_{1}$ and $\beta_{3}$. This concludes the proof. 
Table 4A

Residual Means (Bias) for Various Subadditive Concatenations $g(a, b)$

\begin{tabular}{|c|c|c|c|c|c|c|c|c|}
\hline Group & $\mathrm{CB}$ & SUP & EUC & $\mathbf{M}(9)$ & $\mathbf{M}(\mathbf{M X})$ & $\mathrm{M}(\mathrm{s} 9)$ & AVG & APW \\
\hline \multicolumn{9}{|c|}{ Horizontal Collinears } \\
\hline $\mathrm{AX} 1$ & .24 & -.13 & -.01 & .08 & .06 & .02 & -.20 & .01 \\
\hline $\mathrm{AX} 2$ & .26 & -.15 & -.01 & .08 & & .01 & -.21 & .00 \\
\hline $\mathrm{AX} 3$ & .16 & -.19 & -.07 & .04 & .03 & -.02 & -.24 & -.02 \\
\hline $\mathrm{AX} 4$ & .22 & -.15 & -.02 & .08 & .06 & .02 & -.21 & .01 \\
\hline AX5 & .25 & -.19 & -.04 & .03 & .01 & -.05 & -.25 & -.05 \\
\hline HW & .21 & -.14 & -.02 & .06 & .05 & -.00 & -.21 & .01 \\
\hline $\mathrm{X}$ & .40 & -.01 & .13 & .14 & .11 & .05 & -.12 & .05 \\
\hline \multicolumn{9}{|c|}{ Vertical Collinears } \\
\hline $\mathrm{AX} 1$ & .37 & -.14 & .04 & .06 & .04 & -.04 & -.20 & -.03 \\
\hline $\mathrm{AX} 2$ & .34 & -.12 & .05 & .11 & .07 & .02 & -.18 & .02 \\
\hline $\mathrm{AX3}$ & .27 & -.15 & -.01 & .06 & .04 & -.02 & -.23 & -.03 \\
\hline $\mathrm{AX} 4$ & .29 & -.15 & .01 & .07 & .04 & -.01 & -.21 & -.01 \\
\hline AX5 & .25 & -.19 & -.04 & .03 & .01 & -.05 & -.25 & -.05 \\
\hline HW & .34 & -.09 & .06 & .11 & .09 & .03 & -.17 & .03 \\
\hline$X$ & .43 & .05 & .17 & .18 & .15 & .10 & -.09 & .07 \\
\hline \multicolumn{9}{|c|}{ Shape Diagonal } \\
\hline $\mathrm{AX} 1$ & 1.5 & .76 & 1.1 & .91 & .87 & .74 & .73 & .83 \\
\hline $\mathrm{AX} 2$ & 1.3 & .65 & .91 & .84 & .78 & .71 & .62 & .76 \\
\hline AX3 & 1.5 & .77 & 1.1 & .88 & .84 & .72 & .73 & .82 \\
\hline $\mathrm{AX} 4$ & 1.4 & .67 & .98 & .84 & .78 & .67 & .66 & .76 \\
\hline AX5 & 1.5 & .73 & 1.0 & .86 & .81 & .69 & .70 & .79 \\
\hline HW & .93 & .13 & .45 & .25 & .21 & .07 & .11 & .19 \\
\hline$X$ & 1.5 & .75 & 1.1 & .87 & .80 & .71 & .73 & .82 \\
\hline
\end{tabular}

Area Diagonal

\begin{tabular}{llrrrrrrr} 
AX1 & .31 & -.09 & .05 & .15 & .14 & .09 & -.14 & .08 \\
AX2 & .24 & -.18 & -.02 & .07 & .04 & -.00 & -.21 & .00 \\
AX3 & .16 & -.15 & -.05 & .05 & .03 & .00 & -.20 & .02 \\
AX4 & .21 & -.17 & -.03 & .07 & .05 & .01 & -.20 & .02 \\
AX5 & .30 & -.15 & .03 & .09 & .07 & .02 & -.17 & .03 \\
HW & .17 & -.20 & -.06 & .04 & .03 & -.02 & -.23 & -.01 \\
X & .17 & .04 & .09 & .16 & .13 & .15 & .01 & .20 \\
\hline
\end{tabular}

Note-CB $=$ city-block $;$ SUP $=$ supremum $;$ EUC $=$ Euclidean; $\mathbf{M}(9)=$ metric for bounded responses, with 9 being the upper bound of the response scale; $\mathbf{M}(\mathbf{M X})=$ same as $\mathbf{M}(9)$, but with upper bound replaced by the maximal response of the subject; $M(s 9)=$ same as $M(9)$, but with a reduced upper bound $s 9 ;$ AVG $=$ average; $A P W=$ average power.

The main difference between the saliency metric defined by the four-parameter model and other metrics, besides the subadditivity, is its lack of translational invariance; that is, the same intradimensional segments produce different distances according to the position of stimuli in the space. In Equations 17A, 17B, and 18A, the terms involving the actual coordinates, instead of the segmental differences, express the lack of invariance, generate the subadditivity predictions, and suggest an expression for contextual effects in similarity judgments.

Contextual effects on dissimilarity judgments have been studied by Krumhansl (1978) and Corter (1987), following Parducci's range-frequency theory of contextual effects in unidimensional categorical ratings (Birnbaum, 1974; Parducci, 1965). Parducci considers that a subject's actual judgments reflect a compromise between two principles: (1) the range principle, whereby subjects use the categories to subdivide the psychological range, which is defined as the difference between the two extreme values of the stimuli that form the context for judgment, into subranges corresponding to each category; and (2) the frequency principle, whereby a subject also tends to use each category for a fixed proportion of his or her judgments.

Parducci postulates that categorical judgments are affected by the frequencies of stimulus presentation. For a geometric model of similarities, Krumhansl (1978) equates stimulus frequencies with the spatial density in the regions surrounding any two stimuli. Her distancedensity model predicts that if two stimuli occupy a spatially relatively less dense region of the stimulus domain, they will be seen as more similar than two other stimuli that differ an equivalent amount but lie in a more spatially dense region of the domain. Corter (1987) did not find empirical support for this kind of contextual effect.

Table 4B

\begin{tabular}{|c|c|c|c|c|c|c|c|c|}
\hline Group & $\mathrm{CB}$ & SUP & EUC & $\mathbf{M ( 9 )}$ & $\mathbf{M}(\mathbf{M X})$ & $M(s 9)$ & AVG & APW \\
\hline \multicolumn{9}{|c|}{ Horizontal Collinears } \\
\hline AX1 & + & - & & & & & - & \\
\hline $\mathbf{A X 2}$ & + & - & & & & & - & \\
\hline AX3 & + & - & & & & & - & \\
\hline AX4 & + & - & & & & & - & \\
\hline AX5 & + & - & & & & & - & \\
\hline HW & + & - & & & & & - & \\
\hline $\mathbf{X}$ & + & & + & + & + & & - & \\
\hline \multicolumn{9}{|c|}{ Vertical Collinears } \\
\hline AX1 & + & - & & & & & - & \\
\hline $\mathrm{AX} 2$ & + & - & & + & & & - & \\
\hline $\mathrm{AX} 3$ & + & - & & & & & - & \\
\hline AX4 & + & - & & & & & - & \\
\hline AX5 & + & - & & & & & - & \\
\hline HW & + & & & + & & & - & \\
\hline $\mathbf{X}$ & + & & + & + & + & & & \\
\hline \multicolumn{9}{|c|}{ Shape Diagonal } \\
\hline AX1 & + & + & + & + & + & + & + & + \\
\hline $\mathrm{AX} 2$ & + & + & + & + & + & + & + & + \\
\hline $\mathrm{AX} 3$ & + & + & + & + & + & + & + & + \\
\hline $\mathrm{AX} 4$ & + & + & + & + & + & + & + & + \\
\hline AX5 & + & + & + & + & + & + & + & + \\
\hline HW & + & + & + & + & + & & + & + \\
\hline$X$ & + & + & + & + & + & + & + & + \\
\hline \multicolumn{9}{|c|}{ Area Diagonal } \\
\hline $\mathrm{AX} 1$ & + & & & + & + & & - & \\
\hline $\mathrm{AX} 2$ & + & - & & & & & - & \\
\hline $\mathrm{AX3}$ & + & - & & & & & - & \\
\hline $\mathrm{AX} 4$ & + & - & & & & & - & \\
\hline AX5 & + & - & & & & & - & \\
\hline HW & + & - & & & & & - & \\
\hline $\mathrm{x}$ & + & & & + & + & + & & + \\
\hline
\end{tabular}

Note-Note-CB = city-block; SUP = supremum; EUC = Euclidean; $M(9)=$ metric for bounded responses, with 9 being the upper bound of the response scale; $M(M X)=$ same as $M(9)$, but with upper bound replaced by the maximal response of the subject; $M(s 9)=$ same as $M(9)$, but with a reduced upper bound s9; AVG = average; $A P W=$ average power. Table shows bias patterns of means for residuals $r=g(a, b)-$ $c$ : Blank $=\mid$ mean $r \mid<.1 .+=$ mean $r>.1 .-=$ mean $r<.1$. 
Table 5

Residual SSQ Relative to SSQ Left by the City-Block Metric (Fit) for Various Subadditive Concatenations $g(a, b)$

\begin{tabular}{|c|c|c|c|c|c|c|c|c|c|c|}
\hline Group & CB & SUP & EUC & $\mathbf{M}(9)$ & $\mathbf{M}(\mathbf{M X})$ & M(s9) & AVG & APW & SSQ(CB) & $\Lambda$ \\
\hline \multicolumn{11}{|c|}{ Horizontal Collinears } \\
\hline AX1 & 1.0 & .35 & .19 & .18 & .15 & .11 & .57 & .11 & .09 & 2 \\
\hline $\mathrm{AX} 2$ & 1.0 & .35 & .24 & .23 & .16 & .14 & .55 & .14 & .12 & \\
\hline $\mathrm{AX} 3$ & 1.0 & 1.1 & .48 & .39 & .37 & .37 & 1.5 & .41 & .06 & \\
\hline $\mathrm{AX} 4$ & 1.0 & .50 & .22 & .26 & .23 & .17 & .81 & .19 & .07 & \\
\hline AX5 & 1.0 & .53 & .23 & .17 & .13 & .19 & .83 & .20 & .10 & \\
\hline HW & 1. & .34 & .20 & .17 & .16 & .10 & .57 & .09 & .09 & 1 \\
\hline $\mathrm{X}$ & 1.0 & .16 & .34 & .21 & .19 & .13 & .30 & .26 & .35 & 2 \\
\hline \multicolumn{11}{|c|}{ Vertical Collinears } \\
\hline $\mathrm{AX} 1$ & 1.0 & .21 & .18 & .12 & .10 & .10 & .32 & .09 & .20 & \\
\hline $\mathrm{AX} 2$ & 1.0 & .20 & .13 & .17 & .1 & .09 & .31 & .09 & .15 & 27 \\
\hline $\mathrm{AX} 3$ & 1.0 & .38 & .30 & .22 & .20 & .17 & .58 & .19 & .14 & 27 \\
\hline AX4 & 1.0 & .30 & .20 & .14 & .11 & .09 & .43 & .09 & .14 & 27 \\
\hline AX5 & 1.0 & .16 & .19 & .15 & .11 & .09 & .21 & .09 & .25 & 27 \\
\hline HW & 1.0 & .14 & .12 & .14 & .13 & .06 & .24 & .07 & .15 & 18 \\
\hline $\mathrm{X}$ & 1.0 & .21 & .38 & .24 & .22 & .15 & .29 & .27 & .39 & 27 \\
\hline \multicolumn{11}{|c|}{ Shape Diagonal } \\
\hline AX1 & 1.0 & .25 & .49 & .36 & .32 & .24 & .23 & .30 & 2.3 & \\
\hline $\mathrm{AX} 2$ & 1.0 & .25 & .49 & .40 & .35 & .28 & .23 & .33 & 1.8 & \\
\hline $\mathrm{AX} 3$ & 1.0 & .26 & .49 & .33 & .30 & .22 & .23 & .29 & 2.4 & \\
\hline $\mathrm{AX4}$ & 1.0 & .23 & .47 & .34 & .30 & .22 & .21 & .28 & 2.1 & \\
\hline AX5 & 1.0 & .24 & .48 & .32 & .29 & .21 & .22 & .28 & 2.3 & 9 \\
\hline HW & 1.0 & .07 & .29 & .10 & .08 & .04 & .08 & .08 & 1.0 & \\
\hline $\mathrm{X}$ & 1.0 & .25 & .49 & .32 & .28 & .21 & .24 & .29 & 2.5 & 9 \\
\hline \multicolumn{11}{|c|}{ Area Diagonal } \\
\hline AX1 & 1.0 & .22 & .16 & .33 & .30 & .21 & .32 & .21 & .12 & 9 \\
\hline $\mathrm{AX} 2$ & 1.0 & .72 & .32 & .42 & .36 & .44 & .90 & .45 & .09 & \\
\hline $\mathrm{AX} 3$ & 1.0 & .96 & .54 & .54 & .46 & .53 & 1.2 & .59 & .06 & 9 \\
\hline $\mathrm{AX} 4$ & 1.0 & .70 & .26 & .27 & .24 & .24 & .80 & .24 & .07 & 9 \\
\hline AX5 & 1.0 & .23 & .05 & .11 & .09 & .07 & .30 & .08 & .11 & 9 \\
\hline HW & 1.0 & .90 & .37 & .27 & .28 & .28 & 1.2 & .31 & .06 & 6 \\
\hline $\mathrm{X}$ & 1.0 & .25 & .37 & .84 & .70 & .76 & .09 & 1.2 & .04 & 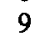 \\
\hline
\end{tabular}

Number of times each metric placed among first three in each row:

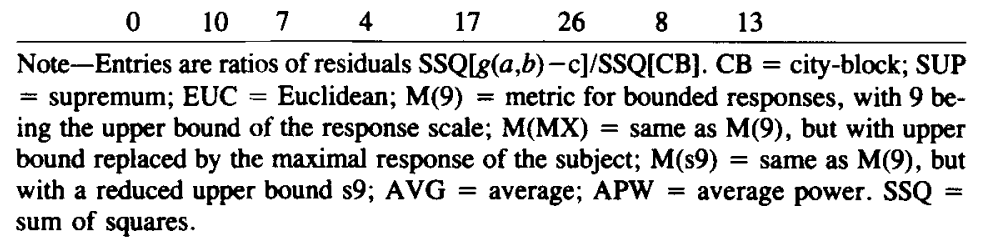

For a saliency metric, the prediction will be that pairs of stimuli with similar intradimensional segments located in the "lower end" of the space (close to the $X_{\text {MIN }}$ and $Y_{\text {MIN }}$, see Figure 2) will be considered less dissimilar than those located at the "upper end" of the space (close to $X_{\text {MAX }}$ and $Y_{\text {MAX }}$; Figure 2).

\section{Saliency Metric and Changes in Response Strategy}

The second question raised by the fits of the fourparameter psychophysical maps was why the ordering of the weights did not agree with previous findings. The plausibility of the saliency metric interpretation of the fourparameter psychophysical maps depends, as we have seen, on the differential weighing of the larger and smaller coordinates on each dimension; that is, the weight for the larger coordinate should be larger than the weight for the smaller coordinate. However, the empirically obtained psychophysical maps point toward the opposite conclusion: In four of the AX groups, the larger eccentricity coordinate had the smaller weight.

In the AX model, the eccentricity variable $X$ was defined as the mean absolute value of the difference between the height and width of a given rectangle, $X=|H-W| / 2$. Thus, $X$ is zero for squares, and it has the same value for rectangles, differing only by a $90^{\circ}$ rotation (a schematic representation to help in the discussion is given in Figure 4). The AX model distinguished only six different stimuli: three squares (Stimuli 1, 5, and 9), and three couples of rotated rectangles (Stimuli 2 and 4, 6 and 8, and 3 and 7). These six stimuli define a total of 15 comparisons, 3 involving only rectangles, 3 involving only squares, and 9 involving both squares and rectangles.

We mentioned before that for individuals following an AX strategy, the pairs that only differ by a $90^{\circ}$ rotation may define a class that may call for a simpler strategy 
Table 6

Fit Indices for Four-parameter Psychophysical Maps of Rectangles, for HW and AX Model, with and without $\tanh ^{-1}$ Transformation

\begin{tabular}{|c|c|c|c|c|c|c|c|c|}
\hline \multirow[b]{3}{*}{ Group } & \multicolumn{4}{|c|}{$\begin{array}{c}\text { Relative } \\
\text { Dissimilarities } d^{*}\end{array}$} & \multicolumn{4}{|c|}{$\begin{array}{c}\text { MBR Transformation } \\
\tanh ^{-1}\left(d^{*}\right)\end{array}$} \\
\hline & \multicolumn{2}{|c|}{ HW Model } & \multicolumn{2}{|c|}{ AX Model } & \multicolumn{2}{|c|}{ HW Model } & \multicolumn{2}{|c|}{ AX Mode } \\
\hline & eta $^{2}$ & $r$ & $\mathrm{eta}^{2}$ & $r$ & $\mathrm{eta}^{2}$ & $r$ & eta $^{2}$ & $r$ \\
\hline $\mathbf{A X} 1$ & .86 & .17 & .95 & .78 & .81 & .18 & .92 & .82 \\
\hline $\mathrm{AX} 2$ & .88 & .15 & .97 & .85 & .84 & .16 & .96 & .88 \\
\hline AX3 & .85 & .19 & .95 & .79 & .80 & .22 & .91 & .81 \\
\hline AX4 & .88 & .21 & .96 & .79 & .83 & .24 & .93 & .81 \\
\hline AX5 & .88 & .12 & .96 & .80 & .85 & .13 & .95 & .84 \\
\hline All $\mathbf{A X}$ & .87 & .16 & .96 & .81 & .83 & .18 & .94 & .84 \\
\hline HW & .96 & .66 & .94 & .33 & .93 & .72 & .89 & .34 \\
\hline $\mathbf{X}$ & .77 & .10 & .94 & .84 & .73 & .08 & .93 & .84 \\
\hline
\end{tabular}

Note-All fits are based on 36 observed average dissimilarities ratings in each group. The models are fitted by least squares without intercept, thus eta ${ }^{2}$ is given by: eta ${ }^{2}=$ [(Uncorrected) Model Sum of Squares]/ [(Uncorrected) Total Sum of Squares]. The values of $r$ correspond to the correlation between the predictions from the (no intercept) model and the observed values. The square of $r$ is equivalent to the $R^{2}$ indexthat is, the least squares fit of the models with intercept. Let $d^{* \prime}=b_{1} W_{L}$ $-b_{2} W_{S}+b_{3} H_{L}-b_{4} H_{S}$, the prediction from one of the models; then $r^{2}$ gives the least squares fit for the model $d^{*}=a_{0}+a_{1} d^{* \prime}=a_{0}+$ $a_{1}\left(b_{1} W_{L}-b_{2} W_{S}+b_{3} H_{L}-b_{4} H_{S}\right)=a_{0}+\left(a_{1} b_{1}\right) W_{L}-\left(a_{1} b_{2}\right) W_{S}+$ $\left(a_{1} b_{3}\right) H_{L}-\left(a_{1} b_{4}\right) H_{S}$.

than the rest of stimulus pairs. We can elaborate this idea following the distinction between squares and rectangles implied by the eccentricity index and illustrated in Figure 4.

Categorical variables have been used before in the multidimensional scaling literature to partition the stimulus set, and to indicate use of different strategies (Torgerson, $1958,1965)$. In 1965, Torgerson revised this idea in terms of similarity as a basic, perhaps perceptual, relation between instances of a multidimensional attribute and similarity as a cognitive relation between stimuli varying on several attributes.

The effect of different strategies on response times has also been studied. In studies of redundancy gain and orthogonal interference, in which pairs of rectangles were presented one at a time (Dykes \& Cooper, 1978), response time was faster when both height and width varied together in such a way that the task could be redefined as consisting of the comparison of shapes or sizes.

The simplest way to cope with the dissimilarity rating task would be to consider only the two categories of square and rectangle, and to assign minimum dissimilarity when both stimuli in a pair come from the same category, and maximum dissimilarity when they come from different categories.

For the stimulus design used, when both stimuli in the pair are from the same category, their difference (regardless of rotation) can be described mostly in terms of size. Therefore, following Dykes and Cooper's (1978) findings, response times would be smaller for stimuli coming from the same category.
The expectation that square-rectangle pairs may produce average ratings and average response times different from those produced by rectangle-rectangle pairs was supported by the results in Table 7. This gives the average relative dissimilarity response and the average response time per pair for square-rectangle pairs (18 pairs) and rectangle-rectangle pairs ( 15 pairs plus the 3 squaresquare pairs) for each group. As the table shows, the average dissimilarity was consistently smaller for rectanglerectangle pairs than for square-rectangle pairs. Furthermore, a clear difference in average response time per pair was found: Square-rectangle pairs required longer responses times than rectangle-rectangle pairs did.

If one assumes, as the results in Table 7 suggest, that square-rectangle versus rectangle-rectangle pairs is the relevant partition of the stimulus set, the possible changes in the strategy used to produce a response can be assessed after fitting the psychophysical models to each of the two

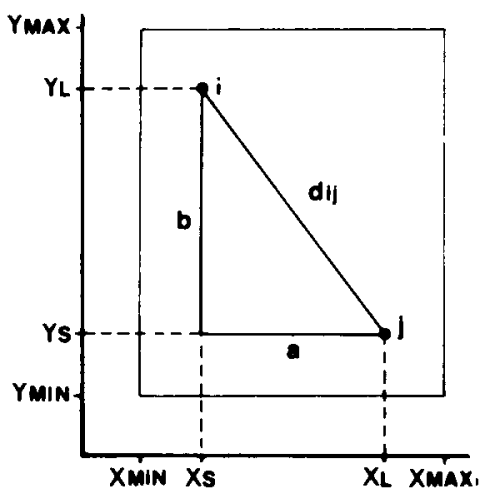

Figure 2. Diagram for the discussion of the saliency metric. In this case, the two stimuli $i$ and $j$ define $X_{L}=X_{j}$ and $Y_{L}=Y_{j}$. $X_{M I N}$, $X_{\text {MAX, }}, Y_{M I N}$, and $Y_{\text {MAX }}$ represent the smallest and largest coordinate projected by the stimulus set.

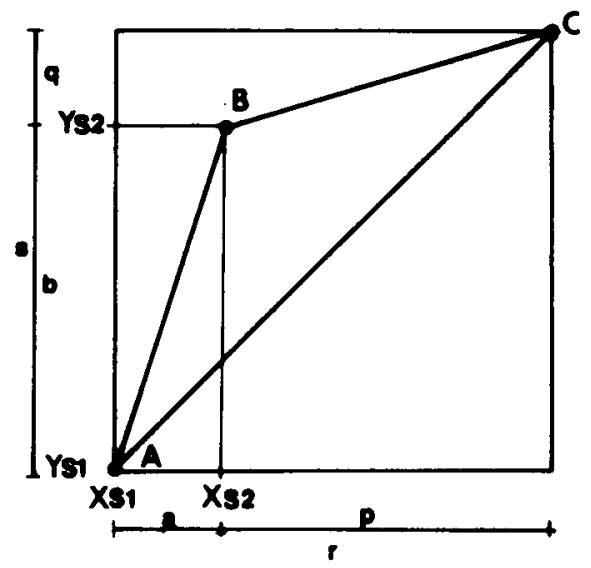

Figure 3. Diagram for the discussion of the triangle inequality property for the saliency metric. Smallest coordinates for $d_{A}$ and $d_{A C}$ are $X_{s 1}$ and $Y_{s 1}$. For $d_{s c}$ the smallest coordinates are $X_{s 2}$ and $Y_{\mathbf{s 2}}$. 


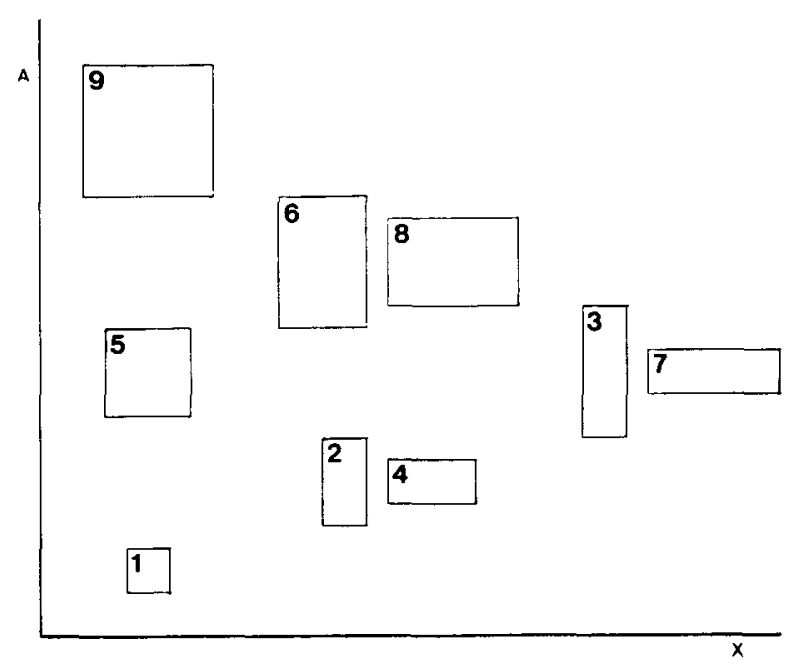

Figure 4. Redefinition of the stimulus layout along the area, $A$, and eccentricity, $X$, dimensions.

different types of pairs. The four-parameter psychophysical maps were fitted separately to rectangle-rectangle (including square-square) and square-rectangle pairs. The results for the best fitting model in each group are shown in Tables $8 \mathrm{~A}$ and $8 \mathrm{~B}$.

For the rectangle-rectangle pairs (Table $8 \mathrm{~A}$ ), the fits followed the same trend as that observed when all 36 pairs were used (Table 6); that is, the AX model gave better fits for the AX subgroups. Thus, for pairs with both elements from the same class of general shape (square-square pairs were included also in this fit), the responses can be expressed in terms of the size and eccentricity of the stimulus. Finally, within the rectangle-rectangle pairs, the fourparameter psychophysical map defines a metric, since now the weights for the eccentricity coordinates follow the ordering required by the saliency metric (see Table $8 \mathrm{~A}$ ).

For the square-rectangle pairs (Table 8B), the HW model fits better than the AX model, with values of eta ${ }^{2}$ and $r$ all exceeding .90 and .80 . The $\mathrm{HW}$ model that directly relates height and width to the dissimilarity responses gives the best fit, even for the groups that were a priori classified as AX groups. However, this model produced poor fits when applied to the subset of rectangle-rectangle pairs.

Most importantly, for all groups, the order of the weights required by the saliency metric (weights for the larger coordinates larger than the weights for the smaller coordinate) is restored by conditioning on the type of stimulus pair.

Taken together, these results suggest that the subjects judged the two classes of pairs differently: (1) For rectangle-rectangle pairs, it is faster to focus on the shape and size of the rectangles; (2) for square-rectangle pairs, the basic strategy is to use height and width, leading to a greater response time. Within each strategy, the concatenation rule can be conceptualized as a subadditive saliency metric. When this distinction between pairs and strategy type is ignored, the ordering of the weights required by the saliency metric is destroyed.

\section{Saliency Metric and Previous Studies}

The dissimilarity judgments for rectangles can be related to the physical dimensions of the stimuli involved. Also, since these judgments are consistently subadditive, they can always be represented by a distance function. Combination of these two characteristics suggests psychophysical maps that define a subadditive saliency metric if the larger coordinates are associated with larger weights. However, in order to demonstrate the proper order of the weights, the present data must be analyzed by type of pair.

The present study differs in two ways from others in which dissimilarity judgment of rectangles was used. First, the physical stimulus design included squares on the diagonal, creating an intuitively appealing, alternative perceptual organization of the stimuli (as in Figure 4). Second, the total number of rectangles involved was small: 9 rectangles that produced 36 different pairs. Schönemann and Lazarte (1987) also used squares on the diagonal of the stimulus design, but their 16 rectangles produced 120 different pairs. On the other hand, Schönemann et al. (1985) used 9 rectangles without squares on the design diagonal, and Borg and Leutner (1983) used 16 rectangles and no squares on the diagonal.

In Schönemann and Lazarte (1987), roughly the same numbers of subjects were classified in the $\mathrm{HW}$ and $\mathrm{AX}$ groups, whereas in the present study, only 1 out of 10 subjects could be classified in the HW group. We speculate that, for stimulus designs that facilitate an alternative structure-squares on the diagonal in our case-the smaller the number of rectangles, the greater will be the tendency to adopt an alternative strategy to the heightwidth strategy.

On the other hand, in Schönemann et al. (1985), the number of stimulus pairs was small, but the stimulus design did not favor any alternative structure; again, approximately half of the subjects preferred the height-width strategy. Thus, it seems that even when the number of stimuli is small, the lack of an obvious alternative organization of the stimuli fosters the reliance on the heightwidth strategy.

Table 7

Average Relative Dissimilarity and Average Response Time (in seconds) Per Pair by Type of Pair

\begin{tabular}{lccccc}
\hline & \multicolumn{2}{c}{$\begin{array}{c}\text { Square-Rectangle } \\
\text { Pairs }\end{array}$} & & \multicolumn{2}{c}{$\begin{array}{c}\text { Rectangle-Rectangle } \\
\text { Pairs }\end{array}$} \\
\cline { 2 - 3 } \cline { 5 - 6 } Group & Dissimilarity & $\begin{array}{c}\text { Response } \\
\text { Time }\end{array}$ & & Dissimilarity & $\begin{array}{c}\text { Response } \\
\text { Time }\end{array}$ \\
\hline AX1 & .68 & 3.22 & & .44 & 2.63 \\
AX2 & .64 & 3.45 & & .44 & 2.98 \\
AX3 & .64 & 4.38 & & .42 & 3.70 \\
AX4 & .63 & 3.56 & & .44 & 3.00 \\
AX5 & .70 & 3.08 & & .49 & 2.83 \\
HW & .64 & 3.21 & & .60 & 2.98 \\
X & .68 & 2.95 & & .24 & 2.75 \\
\hline
\end{tabular}


Table 8A

Four-Parameter Psychophysical Maps for Rectangles Dissimilarity Judgments for 18 Rectangle-Rectangle Pairs, With Saliency Ordering Restored

\begin{tabular}{lrrrr}
\hline Group & & Maps & eta $^{2}$ & $r$ \\
\hline AX1 & $d^{*}$ & $=.12 X_{L}-.07 X_{S}+.19 A_{L}-.17 A_{S}$ & .92 & .78 \\
& $\tanh { }^{-1}\left(d^{*}\right)$ & $=.12 X_{L}-.03 X_{S}+.23 A_{L}-.21 A_{S}$ & .89 & .82 \\
AX2 & $d^{*}$ & $=.13 X_{L}-.07 X_{S}+.20 A_{L}-.18 A_{S}$ & .96 & .86 \\
& $\tanh { }^{-1}\left(d^{*}\right)$ & $=.12 X_{L}-.05 X_{S}+.24 A_{L}-.22 A_{S}$ & .95 & .89 \\
AX3 & $d^{*}$ & $=.12 X_{L}-.01 X_{S}+.20 A_{L}-.20 A_{S}$ & .93 & .80 \\
& $\tanh { }^{-1}\left(d^{*}\right)$ & $=.10 X_{L}-.06 X_{S}+.23 A_{L}-.23 A_{S}$ & .91 & .83 \\
AX4 & $d^{*}$ & $=.11 X_{L}-.02 X_{S}-.18 A_{L}+.16 A_{S}$ & .96 & .83 \\
& $\tanh { }^{-1}\left(d^{*}\right)$ & $=.12 X_{L}-.01 X_{S}-.21 A_{L}+.20 A_{S}$ & .94 & .85 \\
AX5 & $d^{*}$ & $=.16 X_{L}-.09 X_{S}+.20 A_{L}-.17 A_{S}$ & .95 & .81 \\
& $\tanh { }^{-1}\left(d^{*}\right)$ & $=.17 X_{L}-.08 X_{S}+.24 A_{L}-.21 A_{S}$ & .92 & .84 \\
& $d^{*}$ & $=.12 X_{L}-.05 X_{S}+.07 A_{L}-.06 A_{S}$ & .95 & .42 \\
& $\tanh { }^{-1}\left(d^{*}\right)$ & $=.12 X_{L}-.15 X_{S}+.08 A_{L}-.05 A_{S}$ & .92 & .42 \\
H W & $d^{*}$ & $=.07 W_{L}-.03 W_{S}+.08 H_{L}-.05 H_{S}$ & .97 & .69 \\
& $\tanh { }^{-1}\left(d^{*}\right)$ & $=.10 W_{L}-.05 W_{S}+.11 H_{L}-.08 H_{S}$ & .95 & .73
\end{tabular}

Note-The three square-square pairs are included.

Table 8B

Four-Parameter Psychophysical Maps for Rectangles Dissimilarity Judgments for 18 Rectangle-Square Pairs, With Saliency Ordering Restored

\begin{tabular}{|c|c|c|c|}
\hline Group & Maps & $\mathrm{eta}^{2}$ & $r$ \\
\hline $\mathbf{A X 1}$ & $\begin{aligned} d^{*} & =.11 W_{L}-.06 W_{S}+.14 H_{L}-.11 H_{S} \\
\tanh ^{-1}\left(d^{*}\right) & =.17 W_{L}-.12 W_{S}+.22 H_{L}-.22 H_{S}\end{aligned}$ & $\begin{array}{l}.98 \\
.99\end{array}$ & .85 \\
\hline $\mathrm{AX} 2$ & $\begin{aligned} d^{*} & =.10 W_{L}-.06 W_{S}+.12 H_{L}-.08 H_{S} \\
\tanh -1\left(d^{*}\right) & =.15 W_{L}-.11 W_{S}+.17 H_{L}-.14 H_{S}\end{aligned}$ & $\begin{array}{l}.99 \\
.99\end{array}$ & $\begin{array}{l}.88 \\
.90\end{array}$ \\
\hline $\mathrm{AX} 3$ & $\begin{aligned} d^{*} & =.11 W_{L}-.08 W_{S}+.14 H_{L}-.11 H_{S} \\
\tanh -1\left(d^{*}\right) & =.18 W_{L}-.15 W_{S}+.21 H_{L}-.20 H_{S}\end{aligned}$ & $\begin{array}{l}.99 \\
.99\end{array}$ & $\begin{array}{l}.92 \\
.94\end{array}$ \\
\hline $\mathrm{AX} 4$ & $\begin{aligned} d^{*} & =.11 W_{L}-.08 W_{S}-.13 H_{L}+.11 H_{S} \\
\tanh { }^{-1}\left(d^{*}\right) & =.17 W_{L}-.14 W_{S}-.20 H_{L}+.20 H_{S}\end{aligned}$ & $\begin{array}{l}.98 \\
.99\end{array}$ & $\begin{array}{l}.89 \\
.92\end{array}$ \\
\hline AX5 & $\begin{aligned} d^{*} & =.11 W_{L}-.06 W_{S}+.12 H_{L}-.09 H_{S} \\
\tanh { }^{-1}\left(d^{*}\right) & =.18 W_{L}-.12 W_{S}+.19 H_{L}-.17 H_{S}\end{aligned}$ & $\begin{array}{l}.98 \\
.98\end{array}$ & $\begin{array}{l}.78 \\
.84\end{array}$ \\
\hline$X$ & $\begin{aligned} d^{*} & =.20 X_{L}+.10 A_{L}-.03 A_{S} \\
\tanh { }^{-1}\left(d^{*}\right) & =.28 X_{L}+.12 A_{L}-.04 A_{S}\end{aligned}$ & $\begin{array}{l}.98 \\
.98\end{array}$ & $\begin{array}{l}.54 \\
.59\end{array}$ \\
\hline HW & $\begin{aligned} d^{*} & =.12 W_{L}-.10 W_{S}+.14 H_{L}-.12 H_{S} \\
\tanh -{ }^{1}\left(d^{*}\right) & =.20 W_{L}-.19 W_{S}+.23 H_{L}-.22 H_{S}\end{aligned}$ & $\begin{array}{l}.98 \\
.98\end{array}$ & $\begin{array}{l}.85 \\
.90\end{array}$ \\
\hline
\end{tabular}

Note-Model AX only has three parameters in Group X.

In our case, the combination of an obvious alternative organization of the stimuli and a small number of stimuli seemed to interact in a special way. The number of stimuli involved was reduced to two groups of clearly distinct figures: three rectangles (regardless of rotation) and three squares (see Figure 4). As we mentioned before, the appropriate order of the weights in the psychophysical maps that define the subadditive saliency metric were recovered when this categorization was taken into account.

The previous reasoning suggests that if the number of stimuli is large, and/or there is not an obvious perceptual organization of the stimuli, the psychophysical maps should tend to show the required ordering of the weights.

To confirm these intuitions about the influence of the characteristics of the physical stimulus design and the number of stimuli, the four-parameter psychophysical maps were fit to the previously reported data (Borg \&
Leutner, 1983; Schönemann et al., 1985; Schönemann \& Lazarte, 1987). Table 9 gives a summary of the reanalyses.

Regardless of the differences in the number of pairs and the actual stimulus sizes used in each study, the fourparameter psychophysical maps produce good fits. Moreover, with the exception of the UP group (see Table 9) in Schönemann et al. (1985), the weights along each dimension follow the ordering predicted by a saliency metric interpretation.

Only the Schönemann and Lazarte (1987) study included squares. However, the number of stimuli was large enough to leave a large number of pairs even after the design was collapsed along the diagonal.

In the Borg and Leutner (1983) study, the same subjects were given the two physical stimulus designs (120 different pairs each): a completely crossed height $\times$ width 
Table 9

Four-Parameter Psychophysical Maps for Rectangles Dissimilarity Judgments Data Reported Previously in the Literature

\begin{tabular}{|c|c|c|c|c|}
\hline Study & & Maps & $\mathrm{eta}^{2}$ & $r$ \\
\hline \multirow[t]{2}{*}{$\begin{array}{l}\text { Schönemann and Lazarte } \\
(1987)^{*}\end{array}$} & $\begin{array}{l}\text { HW } \\
\text { Groups }\end{array}$ & $\begin{aligned} d^{*} & =.07 W_{L}-.03 W_{S}+.09 H_{L}-.08 H_{S} \\
\tanh -1\left(d^{*}\right) & =.09 W_{L}-.05 W_{S}+.13 H_{L}-.14 H_{S}\end{aligned}$ & $\begin{array}{l}.98 \\
.98\end{array}$ & $\begin{array}{l}.91 \\
.94\end{array}$ \\
\hline & $\begin{array}{c}\text { AX } \\
\text { Groups }\end{array}$ & $\begin{aligned} d^{*} & =.10 X_{L}-.07 X_{S}+.14 A_{L}-.10 A_{S} \\
\tanh -1\left(d^{*}\right) & =.13 X_{L}-.10 X_{S}+.20 A_{L}-.17 A_{S}\end{aligned}$ & $\begin{array}{l}.97 \\
.96\end{array}$ & $\begin{array}{l}.80 \\
.84\end{array}$ \\
\hline \multirow[t]{3}{*}{$\begin{array}{l}\text { Schönemann, Dorcey and } \\
\text { Kienapple (1985) } \dagger\end{array}$} & $\begin{array}{c}\text { EQ } \\
\text { Group }\end{array}$ & $\begin{aligned} d^{*} & =.08 W_{L}-.06 W_{S}+.20 H_{L}-.17 H_{S} \\
\tanh -1\left(d^{*}\right) & =.12 W_{L}-.10 W_{S}+.30 H_{L}-.30 H_{S}\end{aligned}$ & $\begin{array}{l}.99 \\
.99\end{array}$ & $\begin{array}{l}.92 \\
.96\end{array}$ \\
\hline & $\begin{array}{l}\text { UP } \\
\text { Group }\end{array}$ & $\begin{aligned} d^{*} & =.21 X_{L}-.16 X_{S}-.03 A_{L}+.11 A_{S} \\
\tanh { }^{-1}\left(d^{*}\right) & =.37 X_{L}-.26 X_{S}-.11 A_{L}+.18 A_{S}\end{aligned}$ & $\begin{array}{l}.93 \\
.90\end{array}$ & $\begin{array}{l}.65 \\
.72\end{array}$ \\
\hline & $\begin{array}{l}\text { DOWN } \\
\text { Group }\end{array}$ & $\begin{aligned} d^{*} & =.07 W_{L}-.04 W_{S}+.13 H_{L}-.10 H_{S} \\
\tanh { }^{1}\left(d^{*}\right) & =.08 W_{L}-.06 W_{S}+.18 H_{L}-.15 H_{S}\end{aligned}$ & $\begin{array}{l}.96 \\
.94\end{array}$ & $\begin{array}{l}.72 \\
.71\end{array}$ \\
\hline \multirow[t]{2}{*}{ Borg and Leutner (1983) $\ddagger$} & $\begin{array}{c}\text { HW } \\
\text { Design }\end{array}$ & $\begin{aligned} d^{*} & =.09 W_{L}-.06 W_{S}+.28 H_{L}-.24 H_{S} \\
\tanh -1\left(d^{*}\right) & =.13 W_{L}-.12 W_{S}+.45 H_{L}-.42 H_{S}\end{aligned}$ & $\begin{array}{l}.98 \\
.98\end{array}$ & $\begin{array}{l}.90 \\
.94\end{array}$ \\
\hline & $\begin{array}{c}\text { AS } \\
\text { Design }\end{array}$ & $\begin{aligned} d^{*} & =.10 W_{L}-.08 W_{S}+.17 H_{L}-.14 H_{S} \\
\tanh ^{-1}\left(d^{*}\right) & =.14 W_{L}-.14 W_{S}+.29 H_{L}-.27 H_{S}\end{aligned}$ & $\begin{array}{l}.98 \\
.97 \\
\end{array}$ & $\begin{array}{l}.90 \\
.92 \\
\end{array}$ \\
\hline
\end{tabular}

Note-Only the best fit is reported in each case. *From their Table 2. Sixteen rectangles, 120 different pairs. HW group $N=18$, AX group $N=17$. One completely crossed $\mathrm{H} \times \mathrm{W}$ design: heights and widths of $2,4,6$, and $8 \mathrm{~cm}$; squares on main diagonal. †From their Table 7. 9 rectangles, 36 different pairs. EQ group $N=20$, UP group $N=9$, DOWN group $N=6$. One completely crossed $\mathrm{H} \times \mathrm{W}$ design: heights between 1.1 and $3.1 \mathrm{~cm}$; widths between 2.7 and $8.1 \mathrm{~cm}$. $¥$ From their Table 2.16 rectangles, 120 different pairs. $N=42$. Two completely crossed designs: $\mathrm{H} \times \mathrm{W}$, heights between .5 and $2.75 \mathrm{~cm}$, widths between 3 and $6.75 \mathrm{~cm} . \mathrm{A} \times \mathrm{S}$ (area $\times$ shape) design: heights between 3 and $7.24 \mathrm{~cm}$, widths between .35 and $4.59 \mathrm{~cm}$.

design, and a completely crossed area $\times$ shape design. Even for the area and shape designs, an HW model still produced the best fit.

\section{DISCUSSION}

Notwithstanding the simplicity of stimuli such as rectangles, there is no unique way to produce dissimilarity ratings. As we expected from our previous studies, and from studies with such different stimuli as sounds (Melara \& Marks, 1990), different people use different strategies to produce their judgments. Some of these strategies take advantage of the peculiarities of the stimuli to simplify the task by reducing the number of rectangles needed to be distinguished. Only the HW groups distinguished among all nine rectangles, while the $\mathrm{AX}$ groups collapsed the design along the diagonal of squares, thus reducing the number of distinguishable stimuli from nine to six (three squares and three rectangles). Finally, the $\mathrm{X}$ group only distinguished between two classes: squares and rectangles.

The presence of systematic individual differences always represents a nuisance for any general model of behavior. Geometric models of dissimilarity have usually been criticized for violating the triangle inequality. However, in agreement with our earlier research, we found again that direct dissimilarity ratings of rectangles were subadditive along all four conceivable coordinate axes and, therefore, satisfied the triangle inequality regardless of the strategy used by a subject. Since, by the nature of the rating task, only violations of the triangle inequality could rule out distance functions as composition rules, the consistent finding of subadditivity means that the observed dissimilarity ratings can always be conceptualized as distances, albeit not Minkowski distances.

Recognizing the plausibility of a subadditive distance function as a representation of dissimilarity ratings does not necessarily imply the existence of some internal spatial representation, nor, of course, does it rule it out. Most dissimilarity studies, within a geometric model, have relied on MDS algorithms that use as input the matrix of judged dissimilarities. As we mentioned before, the a priori assumption on which these algorithms are based is that, after a suitable ad hoc monotonic transformation, the data are distances produced by the location of the stimuli in a dimensional space that reflects the subjective representation of the stimuli.

In this study, we replicated the earlier finding that the dissimilarity ratings can be directly related to the physical height and width dimensions, and we also showed that the psychophysical maps also defined a distance function. The psychophysical maps are simple linear models that relate the observed dissimilarity to physical dimensions of the observed stimulus pair, and they describe a strategy that is a function of pair-by-pair comparisons. There is no implication that a subjective spatial representation of all the stimuli is generated, that the specific pair is located in that psychological space, or that their distance is transformed into a dissimilarity judgment.

In a previous study (Schönemann \& Lazarte, 1987), the psychophysical maps were subjected to a negatively accelerated response function, the $\tanh ^{-1}$ transformation, in order to remove the subadditivity conjectured to be due to the bounded response scale and to improve the fit for an additive representation. Here we have shown that a map relating the direct ratings to the stimulus's physical 
dimensions not only produces equally good fits, but also provides a link between the psychophysical modeling and the subadditivity of the responses. Under the restriction on the ordering of the weights-that is, that the weight of the larger coordinate should not be less than the weight of the smaller coordinate-the psychophysical maps actually define a subadditive distance function. The larger weights for the larger coordinates suggest that subjects focus principally on the more salient characteristics of the stimuli or stimulus pair when producing their judgments. Thus, the appeal of this saliency metric is threefold: (1) It gives a good fit to the data; (2) it explains the observed subadditivity in the ratings; and (3) it provides an insight into the judgment process involved in producing the dissimilarity ratings.

Ironically, the required ordering of the weights was observed in the reanalyses of previous studies, but it was violated by most of the AX subgroups in the present study. This reminded us of a suggestion made by Shepard (1964): An individual does not necessarily have to commit himself to only one strategy to judge dissimilarities. Indeed, a closer analysis of the present data revealed that when the maps are fitted separately to each of the two types of stimulus pairs, the required ordering of the saliency metric weights held. This suggested the idea that individuals could be using different strategies depending on the type of stimulus pair. The idea of different strategies was even more appealing after our analysis of the response times: For rectangle-rectangle pairs, a faster way to produce a response involved a strategy focusing on the shape and size of the rectangles; for square-rectangle pairs, the basic strategy of considering height and width was used, leading to a greater average response time.

The empirical differences between the two types of pairs provides ground for interesting theoretical speculation. For example, we can argue that the distinction between the two types of pairs suggests a two-stage process in which the stimulus pair is first identified as a member of a certain class before producing the numerical rating, which is dominated by the more salient of the coordinates. We can even advance the hypothesis that in cases where the two dimensions involved are easily recognized as varying separately (as in the plant stimuli of Tversky \& Gati, 1982), subjects might use different strategies according to the number of dimensions on which the pair differs. Within each category of pairs, the responses could be modeled by a distance function.

Some general and basic principles seem to permeate the behavior of individuals in a similarity rating situation. In our experience, when facing such an ambiguous task, subjects produce responses that are not random but usually consistent, in the sense that that they are similar when the same pair of stimuli is presented twice. Also, the responses vary according to the stimuli presented.

Frequently, the number of different pairs involved in a dissimilarity rating task is large. Although perfect consistency can be obtained through memorizing the response to each possible pair of stimuli, this mechanism would not be considered seriously by any psychologist. In fact, the attractiveness of similarity models is that they propose strategies for simplifying the task and, therefore, minimize the effort involved. As Schönemann (1990) expressed, most people try to get difficult and boring tasks over with as soon as possible, using only the simplest possible rules, and try not to make fools of themselves.

The psychophysical maps define a simple strategy, a saliency metric, based on a concatenation rule that assigns more importance or saliency to the larger physical coordinates of an observed pair of rectangles. It adequately fits the data and, if the weights-ordering restriction is satisfied, provides a description of the subadditivity in the responses.

Our initial categorization of strategy groups assumed that the same strategy was applied to all pairs. But, even when the psychophysical maps gave a good fit, the subadditivity present in the data was not reflected in the weights. Rectangles have a "default" set of physical dimensions in height and width, but also an attractive "alternative" definition in terms of area and shape. Also, squares, although they are rectangles, are usually considered as making up a different category by themselves. In our case, both the presence of squares on the diagonal and the small number of stimuli seemed to facilitate the use of different sets of dimensions for different types of pairs.

The notion of similarity is pervasive in psychological theory, and, like many other widely used terms, this one means different things to different researchers (Gregson, 1975). As is common in science, the search for generality sacrifices the specificity of the data. A general model for similarity leads to a necessary simplification of the object under study. Unfortunately, sometimes the simplification is so extreme that one of the basic tasks of any experiment-to describe the behavior at hand-is neglected when the purpose is to fit the global model. The typical direct dissimilarity rating task is representative of these general models; it is ambiguous in defining the relevant aspects that need consideration. Not surprisingly, most of the best efforts to test geometric models of similarity use very simple stimuli in an attempt to restrict the potential number of dimensions involved in the subjects' judgments. Although not explicitly stated, we assumed in making our selection of simple stimuli that all the subjects would react in the same and unique way, that is, in accordance with the experimenter's physical design configuration. However, their responses suggest that subjects cope in flexible ways with the rating task.

We found that, regardless of their humble appearance and intrinsic lack of importance, rectangles proved to be more complex than previously thought, illustrating that subjects' behavior shows flexibility and diversity even in seemingly simple tasks.

\section{REFERENCES}

Attneave, F. (1950). Dimensions of similarity. American Journal of Psychology, 63, 516-556.

Birnbaum, M. H. (1974). Using contextual effects to derive psychophysical scales. Perception \& Psychophysics, 15, 89-96. 
Borg, I. \& Leutner, D. (1983). Dimensional models for the perception of rectangles. Perception \& Psychophysics, 34, 257-267.

Carrol, J. D., \& Chang, J. J. (1970). Analysis of individual differences in multidimensional scaling via an $\mathrm{N}$-way generalization of Eckart-Young decomposition. Psychometrika, 35, 283-320.

CORTER, J. E. (1987). Similarity, confusability, and the density hypothesis. Journal of Experimental Psychology: General, 116, 238-249.

DUNN, J. C. (1983). Spatial metrics of integral and separable dimensions. Journal of Experimental Psychology: Human Perception \& Performance, 9, 242-257.

DYKEs, J. R., JR., COOPER, R. G., JR. (1978). An investigation of the perceptual basis of redundancy gain and orthogonal interference for integral dimensions. Perception \& Psychophysics, 23, 36-42.

GARNER, W. R. (1970). The stimulus in information processing. American Psychologist, 25, 350-358.

Garner, W. R., \& Felfoldy, G. L. (1970). Integrality of stimulus dimensions in various types of information processing. Cognitive Psychology, 1, 225-241.

Gregson, R. A. M. (1975). Psychometrics of similarity. New York: Academic Press.

Horan, C. B. (1969). Multidimensional scaling: Combining observations when individuals have different perceptual structures. Psychometrika, 34, 129-165.

HymaN, R., \&eLl, A. (1967). Judgments of similarity and spatial models. Perception \& Psychophysics, 2, 233-248.

Hyman, R., Well, A. (1968). Perceptual separability and spatial models. Perception \& Psychophysics, 3, 161-165.

KRANTZ, D., \& TVERSKY, A. (1975). Similarity of rectangles: An analysis of subjective dimensions. Journal of Mathematical Psychology, 12, 4-34.

KrumhanSL, C. (1978). Concerning the applicability of geometric models to similarity data: The interrelationship between similarity and spatial density. Psychological Review, 85, 445-463.

KRUSKAL, J. B. (1964). Multidimensional scaling by optimizing goodness of fit to a nonmetric hypothesis. Psychometrika, 29, 1-27.

Melara, R. D., \& Marks, L. E. (1990). Perceptual primacy of dimensions: Support for a model of dimensional interaction. Journal of Experimental Psychology: Human Perception \& Performance, 16, 398-414.

ParducCI, A. (1965). Category judgment: A range-frequency model. Psychological Review, 72, 407-418.

Richardson, H. W. (1938). Multidimensional psychophysics. Psychological Bulletin, 53, 659-660. (Abstract)
SchönemanN, P. H. (1972). An algebraic solution for a class of subjective metrics models. Psychometrika, 37, 441-451.

SCHÖNEMANN, P. H. (1977). Similarity of rectangles. Joumal of Mathematical Psychology, 16, 161-165.

SchönemanN, P. H. (1982). A metric for bounded response scales. Bulletin of the Psychonomic Society, 19, 317-319.

SchönemanN, P. H. (1983). Some theory and results for metrics for bounded response scales. Journal of Mathematical Psychology, 27, 311-324.

Schönemann, P. H. (1990). Psychophysical maps for rectangles. In H. G. Geissler (Ed.), Psychophysical explorations of mental structures. Toronto: Hogrefe \& Huber.

Schönemann, P. H., Dorcey, T., \& KienapPle, K. (1985). Subadditive concatenation in dissimilarity judgments. Perception \& Psychophysics, 38, 1-17.

SChönemann, P. H., Lazarte, A. (1987). Psychophysical maps for subadditive dissimilarity ratings. Perception \& Psychophysics, 42 , 342-354.

SHEPARD, R. N. (1962a). The analysis of proximities: Multidimensional scaling with an unknown distance function: 1. Psychometrika, 27, 125-140.

SHEPARD, R. N. (1962b). The analysis of proximities: Multi-dimensional scaling with an unknown distance function: II. Psychometrika, 27, 219-246.

SHEPARD, R. N. (1964). Attention and the metric structure of the stimulus space. Journal of Mathematical Psychology, 1, 54-87.

Torgerson, W. S. (1952). Multidimensional scaling: I. Theory and method. Psychometrika, 17, 401-419.

TORGERSON, W. S. (1958). Theory and methods of scaling. New York: Wiley.

Torgerson, W. S. (1965). Multidimensional scaling of similarity. Psychometrika, 30, 379-393.

TVERSKY, A., GATI, I. (1982). Similarity, separability, and the triangle inequality. Psychological Review, 89, 123-154.

WENDER, K. (1971). A test of independence of dimensions in multidimensional scaling. Perception \& Psychophysics, 10, 30-32.

Wiener-EhrLich, W. K. (1978). Dimensional and metric structures in multidimensional stimuli. Perception \& Psychophysics, 24, 399-414.

(Manuscript received March 13, 1990; revision accepted for publication August 9, 1990.) 